\title{
Selective delivery of low-affinity IL-2 to PD-1+ T cells rejuvenates antitumor immunity with reduced toxicity
}

\author{
Zhenhua Ren, ${ }^{1}$ Anli Zhang, ${ }^{1}$ Zhichen Sun, ${ }^{1,2}$ Yong Liang, ${ }^{1}$ Jianfeng Ye, ${ }^{3}$ Jian Qiao, ${ }^{1}$ Bo Li, ${ }^{3}$ and Yang-Xin Fu ${ }^{1,4}$ \\ 'Department of Pathology, ${ }^{2}$ Department of Pharmacology, Harold C. Simmons Comprehensive Cancer Center, and ${ }^{3}$ Lyda Hill Department of Bioinformatics, UT Southwestern Medical Center, Dallas, Texas, \\ USA. ${ }^{4}$ Department of Basic Medical Science, Tsinghua University, Beijing, China.
}

\begin{abstract}
PD-1 signaling on T cells is the major pathway that limits T cell immunity, but the efficacy of anti-PD-1 therapy has been limited to a small proportion of patients with advanced cancers. We fortuitously observed that anti-PD-1 therapy depends on IL-2 signaling, which raises the possibility that a lack of IL-2 limits anti-PD-1-induced effector T cell expansion. To selectively deliver IL-2 to PD-1+CD8+ tumor-infiltrating lymphocytes (TILs), we engineered a low-affinity IL-2 paired with anti-PD-1 (PD-1-lalL-2), which reduced affinity to peripheral Treg cells but enhanced avidity to PD-1+CD8+ TILs. PD-1-lalL-2 exerted better tumor control and lower toxicity than single or mixed treatments. Mechanistically, PD-1-lalL-2 could effectively expand dysfunctional and tumor-specific CD8+ T cells. Furthermore, we discovered that presumably dysfunctional PD-1+TIM3 ${ }^{+}$TILs are the dominant tumor-specific T cells responding to PD-1-lalL-2. Collectively, these results highlight that PD-1-lalL-2 can target and reactivate tumor-specific TILs for tumor regression as a unique strategy with stronger efficacy and lower toxicity.
\end{abstract}

\section{Introduction}

Increased levels of tumor-infiltrating lymphocytes (TILs) are associated with improved survival in patients with cancer (1-3). AntiPD-1/PD-L1-based cancer immunotherapies have revolutionized the treatment of cancers (4-8), and TIL abundance can be used as a prediction marker for immunotherapy responsiveness $(9,10)$. Even though PD-1/PD-L1 blockade can release the brake on the $\mathrm{T}$ cell response, $\mathrm{T}$ cells are not fully functional and are limitedly expanded in the tumor $(11,12)$. Most patients either fail to respond or develop adaptive resistance after an initial response (13-15). Importantly, the role of $\mathrm{T}$ cell-associated cytokines in the tumor microenvironment for anti-PD-1/PD-L1 responsiveness has not been fully studied. It is possible that additional T cell-driven cytokine therapy might overcome PD-1 therapy resistance.

IL-2 is an important $\mathrm{T}$ cell growth factor for T cell proliferation (16). The IL-2 receptor has 3 subunits-IL-2R $\alpha$ (CD25), IL-2R $\beta$ (CD122), and IL-2R $\gamma$-expressed on T cells and NK cells $(17,18)$. The IL-2R $\alpha$ subunit is mainly expressed on Treg cells. Therefore, low doses of IL-2 treatment lead to the production of more Treg cells than $\mathrm{CD}^{+} \mathrm{T}$ cells $(19,20)$. High-dose IL-2 treatment can overcome Treg-associated IL-2 trapping and allow extra IL-2 to activate TILs for treating metastatic renal cell carcinoma and melanoma (21-24). However, patients who respond to high-dose IL-2 treatment frequently suffer from intolerable toxicities (25), which

\section{Related Commentary: https://doi.org/10.1172/JCI156628}

Authorship note: ZR and AZ contributed equally to this work.

Conflict of interest: The authors have declared that no conflict of interest exists.

Copyright: $\odot$ 2022, Ren et al. This is an open access article published under the terms of the Creative Commons Attribution 4.0 International License.

Submitted: July 26, 2021; Accepted: December 3, 2021; Published: February 1, 2022.

Reference information: / Clin Invest. 2022;132(3):e153604.

https://doi.org/10.1172/JCl153604. limits its clinical use. Extensive efforts have been made to generate mutants that either reduce the binding to IL-2R $\alpha$ on Tregs (26) or increase the binding to IL-2R $\beta$ on effector cells $(27,28)$.

How to target IL-2 to tumor-specific T cells remains a challenge in IL-2 cancer immunotherapy. Most studies focus on using antitumor antigens to bring IL-2 into tumor tissues (29-31). For example, fibronectin, which is expressed abundantly around neovascular structures in tumors, has been applied for treatment (32-34). Several other targets have been assessed for their ability to deliver IL-2 to the tumor site; these include carcinoembryonic antigen (CEA) and fibroblast activation protein (FAP) (35-38). However, these strategies can only bring IL-2 to tumor sites, and strategies to target IL-2 effectively and specifically to intratumoral effector T cells instead of other undesired cells have not been discovered thus far.

Here, we designed a fusion protein (IL-2 linked to an anti-PD-1 antibody) to target TILs, as TILs express more PD- 1 than other cells. To reduce the binding of IL-2 to Tregs, we selected a lowaffinity IL-2 (laIL-2) that has greatly reduced binding to both IL-2R $\alpha$ and IL-2R $\beta$. We linked laIL-2 to an anti-PD-1 antibody (generating PD-1-laIL-2) to increase its avidity to intratumoral $\mathrm{CD}^{+} \mathrm{T}$ cells. PD-1-laIL-2 showed better intratumoral $\mathrm{T}$ cell binding and potent antitumor effects. The use of such fusion proteins can also overcome PD-L1 therapy resistance.

\section{Results}

$P D-1-$ laIL-2 selectively targets intratumoral $C D 8^{+} T$ cells. To test whether the therapeutic effect of anti-PD-1 depends on IL-2 signaling, we blocked the IL-2 pathway with anti-IL-2R $\beta$. The therapeutic effect of anti-PD-1 was totally abolished when anti-IL-2R $\beta$ was given (Figure 1A and Supplemental Table 1), which suggests that IL-2 signaling is important for anti-PD-1 immunotherapy. Therefore, we hypothesized that targeting exogenous IL-2 to PD $-1^{+} \mathrm{T}$ cells may greatly expand PD- 1 blockade-rescued dysfunctional TILs to enhance the therapeutic effect. We examined the 

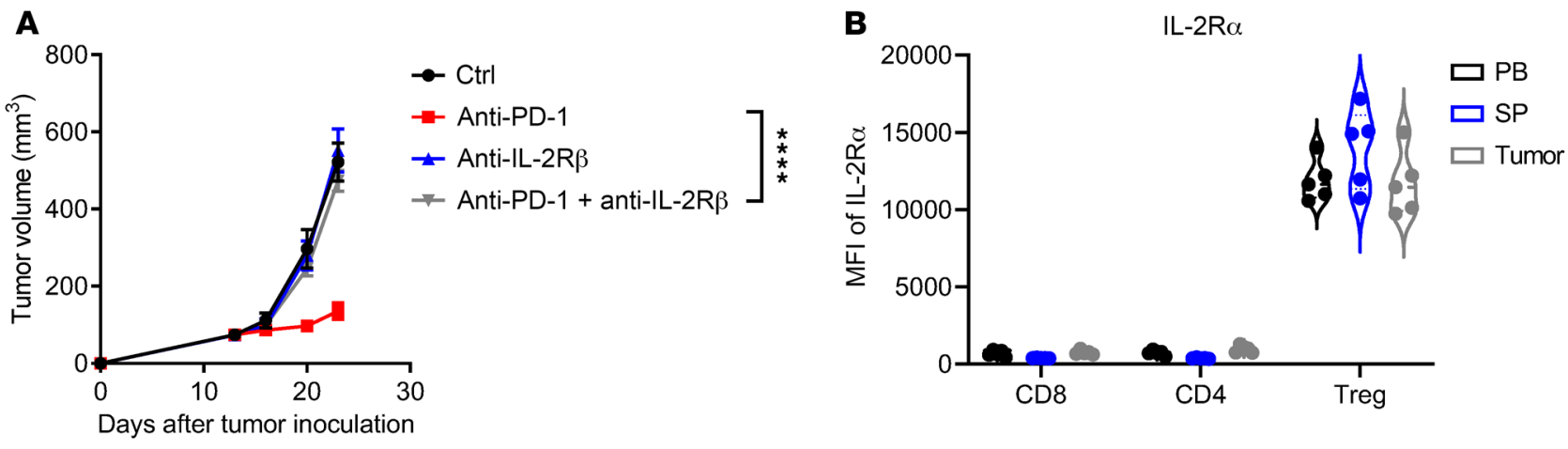

C

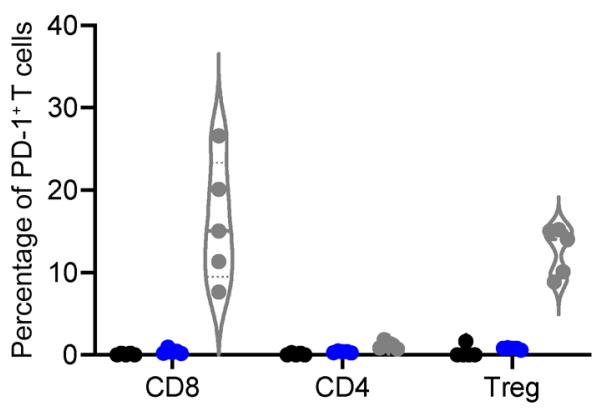

$\mathbf{E}$

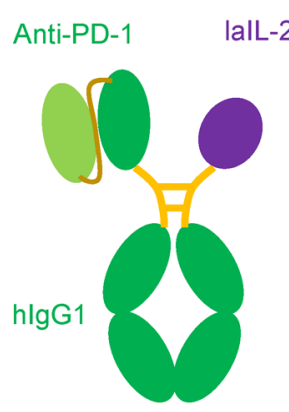

$\mathbf{F}$

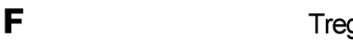

Treg

$\square \mathrm{PB}$

$\square$ SP

$\square$ Tumor

D
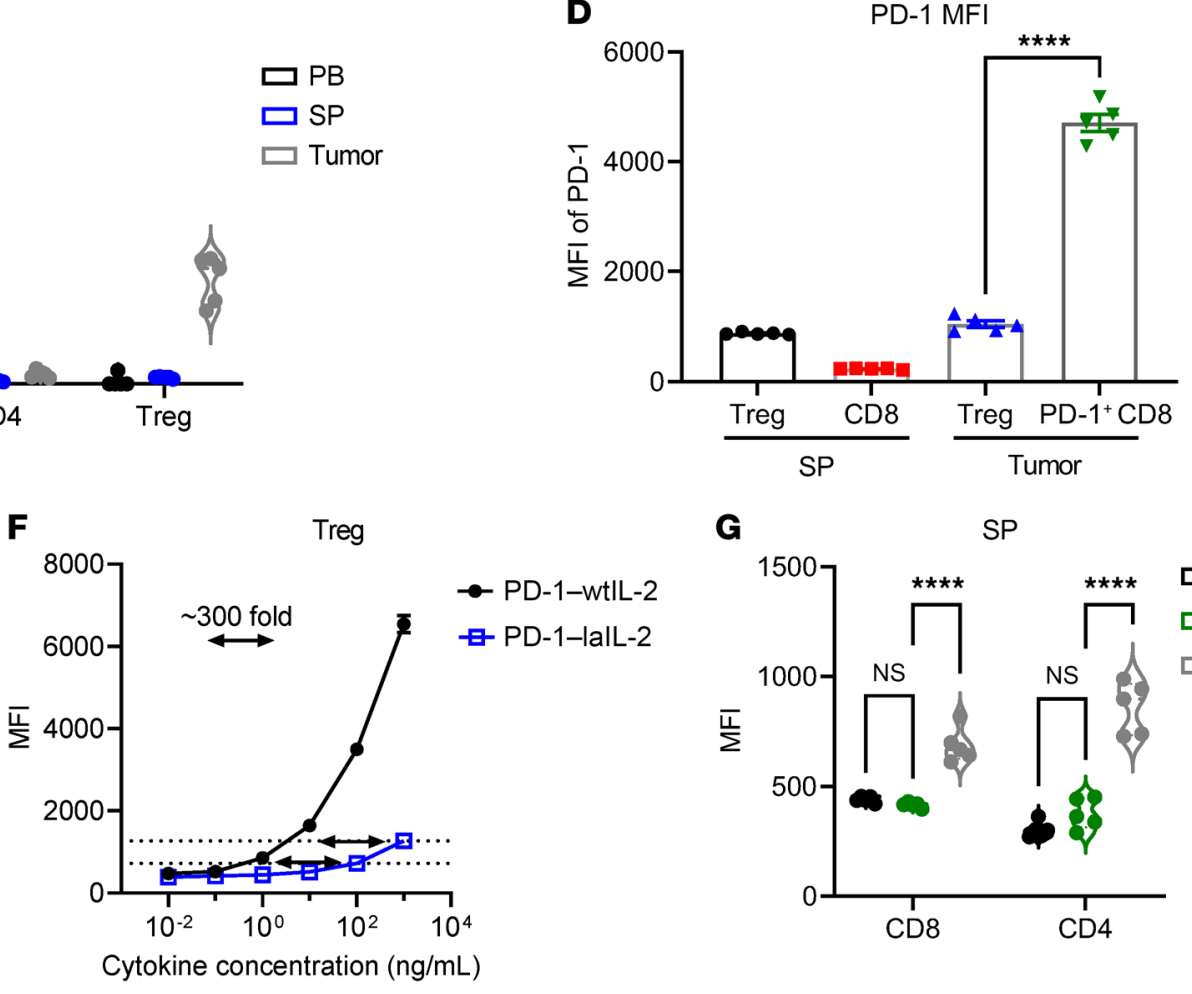

G $\mathrm{SP}$
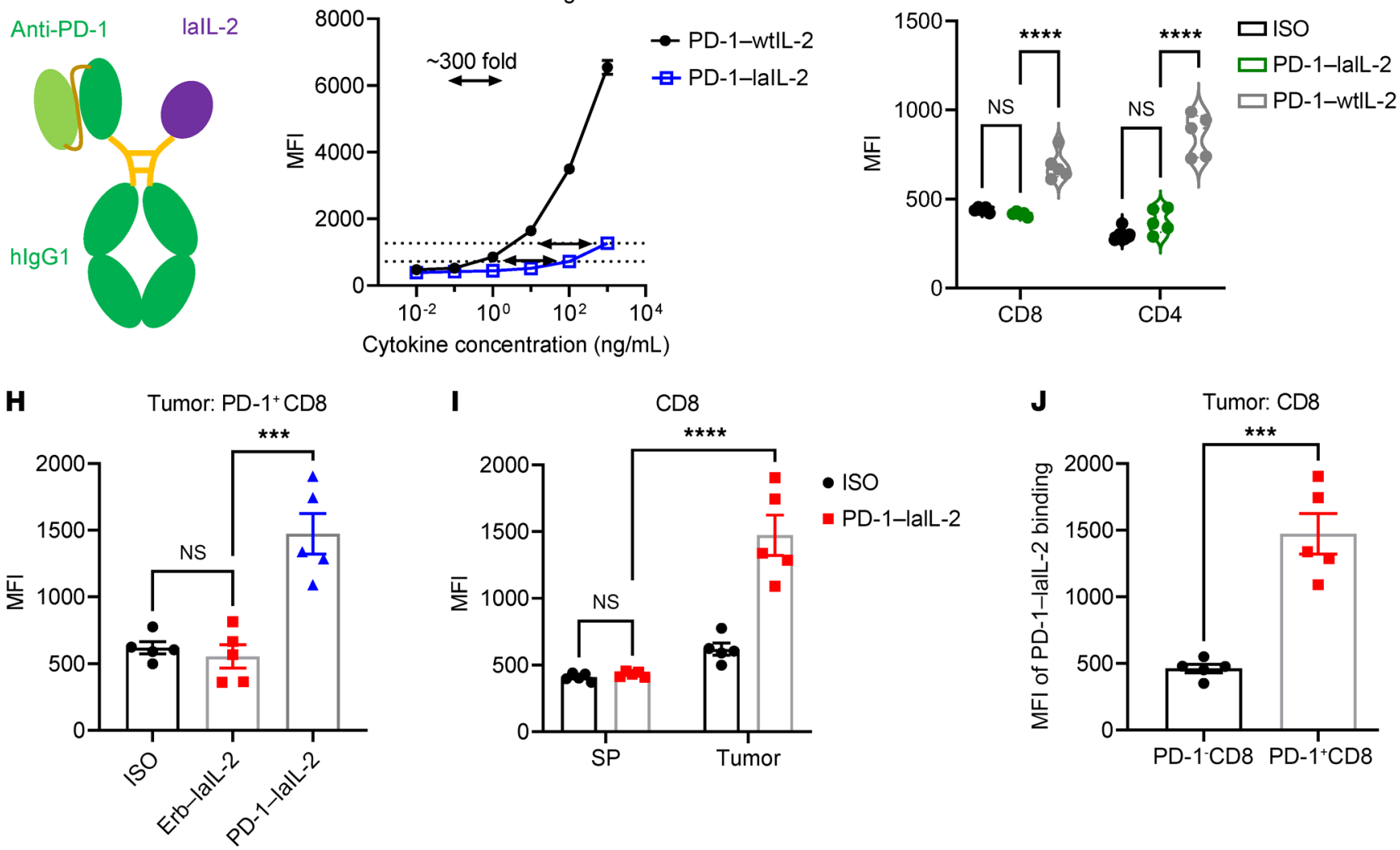

I $\quad \operatorname{CD} 8$
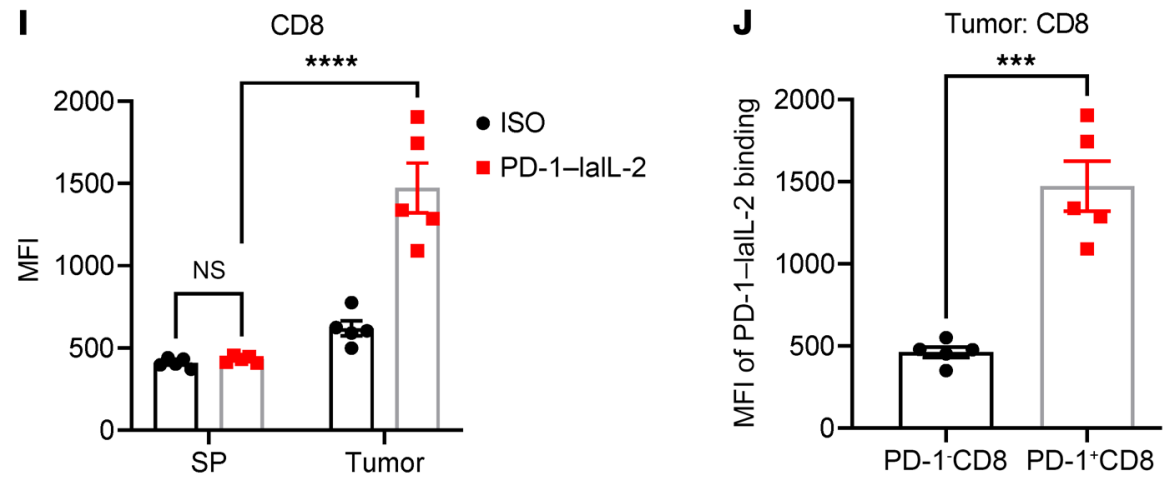
Figure 1. PD-1-lalL-2 selectively targets intratumoral CD8 ${ }^{+} \mathbf{T}$ cells. (A) BALB/c mice ( $n=5$ /group) were inoculated with $2 \times 10^{6}$ A20 tumor cells and were treated with $50 \mu \mathrm{g}$ anti-PD- 1 and/or $200 \mu \mathrm{g}$ anti-IL-2R $\beta$ on day 14. Tumor growth was assessed twice a week. (B) IL-2R $\alpha$ expression on $\mathrm{CD}^{+}, \mathrm{CD}^{+}$, and Treg cells in peripheral blood, spleen, and tumor samples (indicated as PB, SP, and tumor in the figures) from A20 tumor-bearing mice ( $n=5$ /group). (C) Percentages of PD- $1^{+}$T cells in peripheral blood, spleen, and tumor samples from A20 tumor-bearing mice ( $n=5 /$ group). (D) Mean fluorescence intensity (MFI) of PD- 1 on Treg and CD8 ${ }^{+} \mathrm{T}$ cells in the spleen and on Treg and PD-1+CD8 ${ }^{+} T$ cells in the tumors from A20 tumor-bearing mice ( $n=5$ /group). (E) Schematic diagram of the anti-PD-1 $\times$ lalL-2 heterodimer (PD-1-lalL-2). (F and G) PD-1-wtIL-2 and PD-1-lalL-2 bind to Treg (F), CD8 $8^{+}$, and $\mathrm{CD}^{+}(\mathbf{C})$ T cells in the spleen of A20 tumor-bearing mice ( $n=5$ /group). (H) Erb-lalL-2 and PD-1-lalL-2 bind to PD-1+CD8 ${ }^{+}$ T cells in tumors from A20 tumor-bearing mice ( $n=5$ /group). (I) PD-1-lalL-2 binds to $C D 8^{+} T$ cells in the spleen and to $P D-1^{+} C D 8^{+} T$ cells in tumors from A20 tumor-bearing mice ( $n=5 /$ group). (J) PD-1-lalL-2 binds to PD-1-CD8 ${ }^{+}$ and PD-1+CD8 ${ }^{+}$T cells in tumors from A20 tumor-bearing mice $(n=5 /$ group). Data represent mean \pm SEM from 2 to 3 independent experiments. The $P$ value was determined by 2-way ANOVA with Geisser-Greenhouse's correction (A), 1-way ANOVA with Tukey's multiple comparisons test (D and $\mathbf{H}$ ), 2-way ANOVA with Tukey's multiple comparisons test ( $\mathbf{G}$ and $\mathbf{I}$ ), or 2-tailed unpaired $t$ test $(\mathrm{J})$. The normality of the data was confirmed by the Shapiro-Wilk test. ${ }^{* * *} P<0.001,{ }^{* * *} P<0.0001$.

expression of IL-2R $\alpha$ and PD-1 on T cells in tumor-bearing mice. Consistent with previous studies, IL-2R $\alpha$ was mainly expressed on Treg cells (Figure 1B and Supplemental Figure 1A; supplemental material available online with this article; https://doi.org/10.1172/ JCI153604DS1). IL-2R $\beta$ and IL-2R $\gamma$ were universally expressed on all $\mathrm{T}$ cells (Supplemental Figure 1, B and C). Compared with that in the peripheral blood and spleen, there was a higher percentage of $\mathrm{PD}-1^{+} \mathrm{CD} 8^{+} \mathrm{T}$ cells in the tumor (Figure $1 \mathrm{C}$ ). $\mathrm{PD}-1^{+} \mathrm{CD} 8^{+} \mathrm{T}$ cells expressed higher levels of PD-1 than Treg cells in the tumor (Figure 1, C and D). We proposed PD-1 as an appealing target to bring IL-2 to CD $8^{+}$TILs. To reduce the binding of IL- 2 to Treg cells that express IL-2R $\alpha$ and IL-2R $\beta$ and potentially absorb more IL-2, we selected a low-affinity IL-2 (IL-2 R38L F42A, laIL-2) that has greatly reduced binding to both IL-2R $\alpha$ and IL-2R $\beta$ for Treg cells.

Taking advantage of the high expression of PD- 1 on $\mathrm{CD}^{+} \mathrm{T}$ cells among TILs, we engineered PD-1-laIL-2 to increase their avidity to intratumoral $\mathrm{CD} 8^{+} \mathrm{T}$ cells (Figure $1 \mathrm{E}$ and Supplemental Figure 1D). PD-1-laIL-2 had a much lower binding than antiPD-1-linked wild-type IL-2 (PD-1-wtIL-2) to peripheral Treg cells (Figure $1 \mathrm{~F}$ and Supplemental Figure 1E). Moreover, the binding of PD-1-laIL-2 to intratumoral Treg cells was much lower than that of PD-1-wtIL-2 (Supplemental Figure 1F). We also checked the binding of PD-1-laIL-2 to HEK cells that express human IL-2 receptors (HEK-Blue IL-2 cells), and the binding of PD-1-laIL-2 to HEK-Blue IL-2 cells was much lower than that of PD-1-wtIL-2 (Supplemental Figure 1, G and H). Compared with PD-1-wtIL-2, PD-1-laIL-2 also had reduced binding to peripheral $\mathrm{CD}^{+}$and $\mathrm{CD}^{+} \mathrm{T}$ cells, which was almost undetectable and should result in reduced toxicity (Figure 1G). To test whether PD-1 is critical for enhanced avidity to PD-1+ ${ }^{+}$TILs, we compared PD-1-laIL-2 with control antibody-linked laIL-2 (Erb-laIL-2). Indeed, PD-1-laIL-2 bound much more readily to $\mathrm{PD}-1^{+} \mathrm{CD} 8^{+}$TILs than Erb-laIL-2 (Figure $1 \mathrm{H})$. When comparing TILs and peripheral T cells, PD-1-laIL-2 mainly bound to intratumoral PD $-1^{+} \mathrm{CD}^{+} \mathrm{T}$ cells but did not bind to peripheral $\mathrm{CD}^{+} \mathrm{T}$ cells (Figure 1I). Additionally, the binding of PD-1-laIL-2 to intratumoral PD $-1^{+} \mathrm{CD} 8^{+} \mathrm{T}$ cells was much higher than binding to intratumoral PD $-1^{-} \mathrm{CD}^{+} \mathrm{T}$ cells (Figure $1 \mathrm{~J}$ ). Taken together, these findings indicate that PD-1-laIL-2 can selectively target intratumoral CD $8^{+} \mathrm{T}$ cells instead of Treg cells.

PD-1 antibody-armed laIL-2 has enhanced tumor control. We then sought to study whether targeting laIL-2 to intratumoral $\mathrm{CD} 8^{+} \mathrm{T}$ cells has a beneficial outcome in terms of tumor control. Strikingly, a single low dose $(20 \mu \mathrm{g})$ of PD-1-laIL-2 eradicated the established A20 tumors, whereas an equivalent dose of single or combination treatment of anti-PD-1 and Erb-laIL-2 had almost no effect at all (Figure 2A). Importantly, the antitumor effect of PD-1-laIL-2 was not restricted to the A20 tumor model, and PD-1-laIL-2 had much better control of the tumors than anti-PD-1 plus Erb-laIL-2 in the MC38 colon tumor model (Figure $2 \mathrm{~B}$ ) and even the poorly immunogenic Renca renal tumor model (Supplemental Figure 2A). To investigate whether laIL-2 is necessary, we treated tumor-bearing mice with similar molar amounts of PD-1-wtIL-2 and PD-1-laIL-2. PD-1-wtIL-2 had much less tumor control capacity than PD-1-laIL-2 (Figure 2C and Supplemental Figure 2, B and C). These data suggest that reducing the binding of IL-2 to peripheral T cells may allow better tumor targeting. To investigate whether targeting laIL-2 to the tumor site via PD-1 can exert better tumor control than other potential non-T cell targets, we linked laIL-2 to an anti-PD-L1 antibody (PD-L1-laIL-2). PD-L1-laIL-2 had a similar tumor control effect as Erb-laIL-2 or anti-PD-L1 (Figure 2D) and was far less effective than PD-1-laIL-2 (Figure 2E and Supplemental Figure 2C). Therefore, the data suggest that delivering IL- 2 to T cells via anti-PD- 1 is important. Since PD-1/PD-L1 therapy often fails to control more established tumors, we speculated that the enrichment of IL-2 in TILs can amplify antitumor immunity during anti-PD-1/PD-L1 treatment. To avoid the competition between anti-PD-1 and PD-1laIL-2 for PD-1 on T cells, we chose anti-PD-L1 treatment. To test whether PD-1-laIL-2 can overcome anti-PD-L1 therapy resistance, we added PD-1-laIL-2 treatment to a PD-L1 therapy regimen and observed that PD-1-laIL-2 could synergize with anti-PD-L1 therapy in more advanced tumors (Figure 2F). Therefore, PD-1-laIL-2 overcomes resistance to $\mathrm{PD}-\mathrm{L} 1$ blockade therapy. In a humanized mouse model, we observed much better tumor control in the hPD-1-laIL-2 group than in the combination treatment group (Figure $2 \mathrm{G}$ ).

Antitumor efficacy of PD-1-laIL-2 depends on intratumoral CD $8^{+}$ $T$ cells. As IL-2 can provoke the function of NK and T cells (39), we used Rag1/-mice that lack $\mathrm{T}$ and $\mathrm{B}$ cells but have intact NK cells to dissect the contribution of NK and T cells. The therapeutic effect of PD-1-laIL-2 was totally abolished in Rag1 $^{-/-}$mice (Figure 3A), which suggests that NK cells are not sufficient for PD-1-laIL-2-induced tumor control and that $\mathrm{T}$ cells are required for the therapeutic function of PD-1-laIL-2. NK cell depletion had no impact on tumor control by PD-1-laIL-2 (Supplemental Figure 3A), which further shows that NK cells are not required for PD-1-laIL-2 therapy in the current model. To determine which subset of T cells is required, we depleted either $\mathrm{CD}^{+}$or $\mathrm{CD}^{+} \mathrm{T}$ cells before and during PD-1-laIL-2 treatment. $\mathrm{CD}^{+}{ }^{+} \mathrm{T}$ cells were not necessary for PD-1-laIL-2 treatment (Supplemental Figure 3B). However, $\mathrm{CD} 8^{+} \mathrm{T}$ cell depletion completely abolished the therapeutic effect of PD-1-laIL-2 (Figure 3B). Therefore, $\mathrm{CD}^{+} \mathrm{T}$ cells, but not $\mathrm{CD} 4^{+} \mathrm{T}$ cells or NK cells, are required for the therapeutic effect of PD-1-laIL-2 in our model. 
A

A20

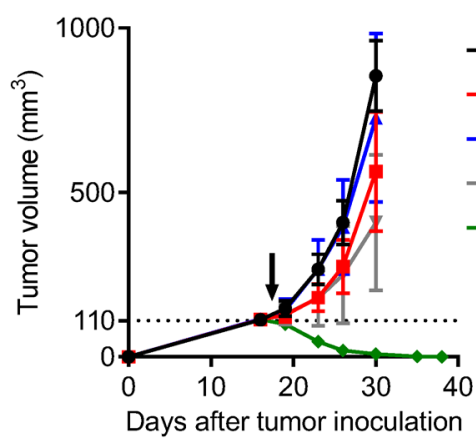

C

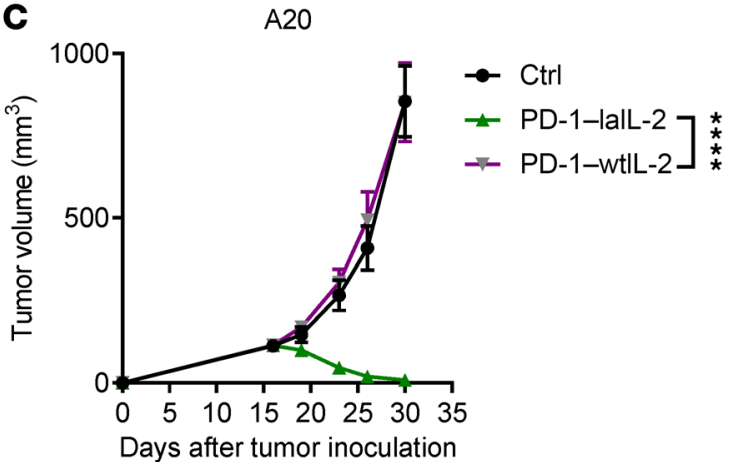

$$
\text { Ctrl }
$$

- Erb-lalL-2

- Anti-PD-1

$\rightarrow$ Anti-PD-1 + Erb-lalL2
$\sim$ PD-1-lalL-2
B

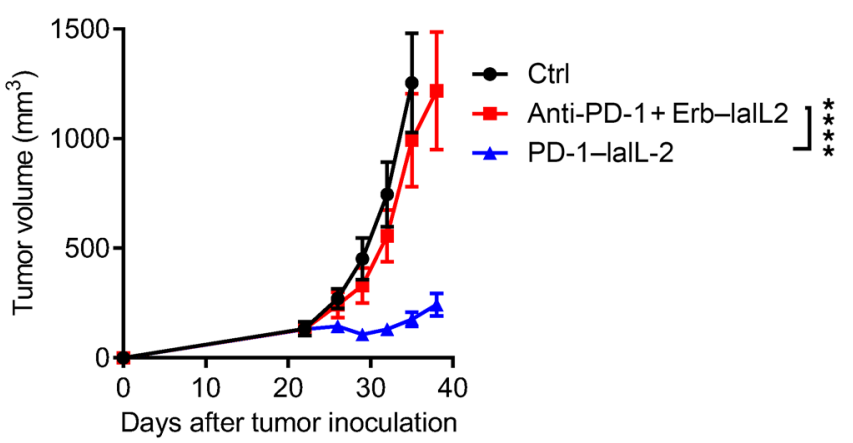

E

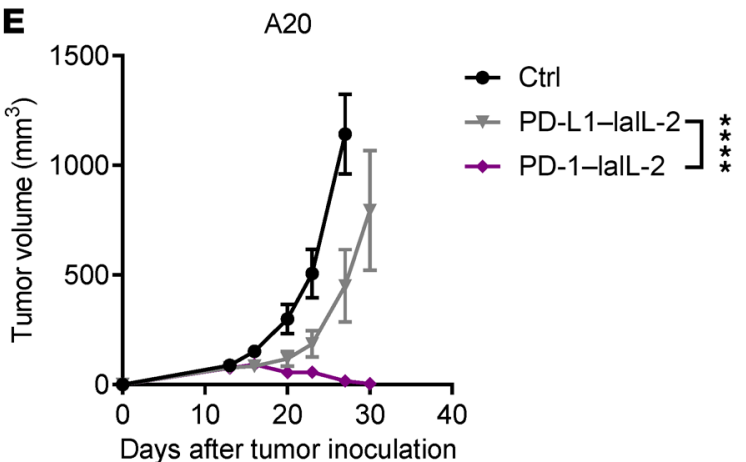

D

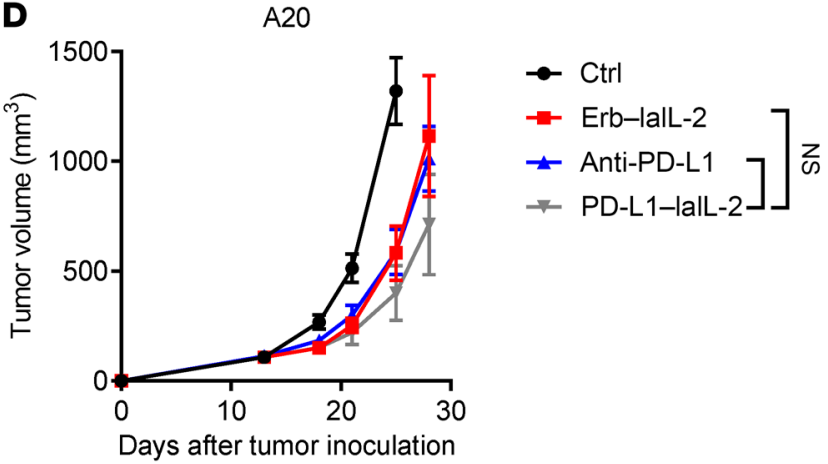

F

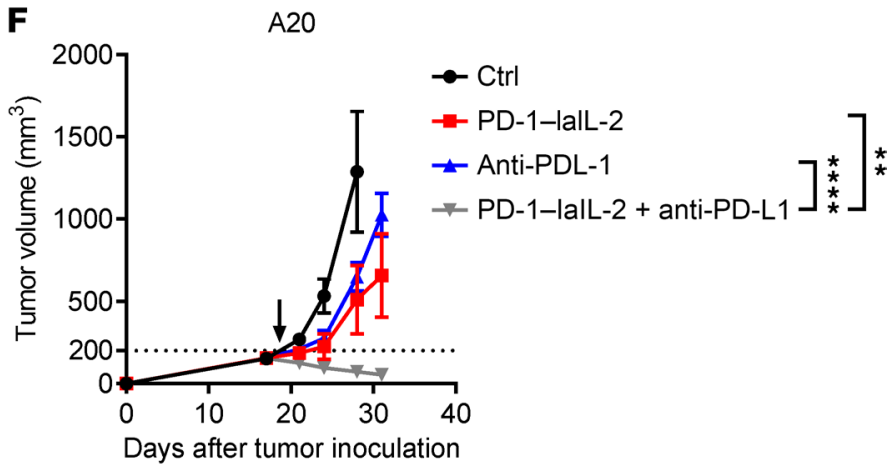

G

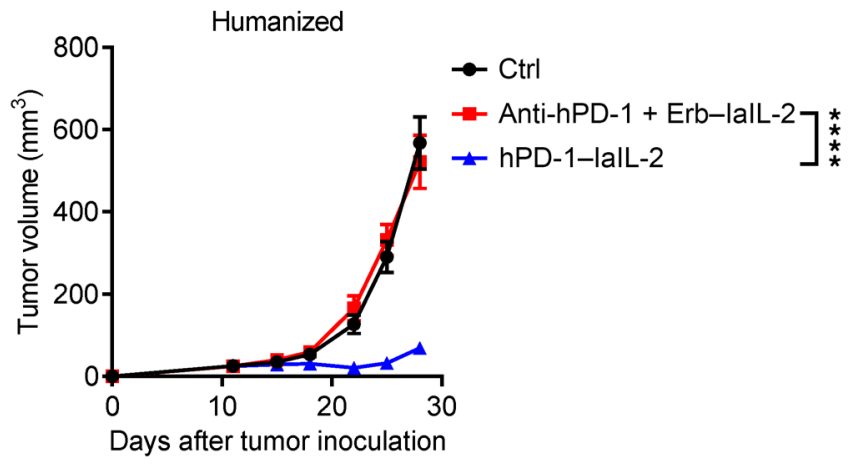

Figure 2. PD-1 antibody-armed lalL-2 has enhanced tumor control. (A) A20 tumor-bearing mice ( $n=5 / g r o u p)$ were treated with equal molar amounts of Erb-lalL-2 (20 $\mu \mathrm{g})$, anti-PD-1 (10 $\mu \mathrm{g})$, or PD-1-lalL-2 $(20 \mu \mathrm{g})$ on day 17. Tumor growth was assessed twice a week. (B) MC38 tumor-bearing mice $(n=5 / g r o u p)$ were treated with equal molar amounts of Erb-lalL-2 $(20 \mu \mathrm{g})$ and anti-PD-1 (10 $\mu \mathrm{g})$ or PD-1-lalL-2 (20 $\mu \mathrm{g})$ on day 21 . Tumor growth was assessed twice a week. (C) A20 tumor-bearing mice ( $n=5$ /group) were treated with $20 \mu \mathrm{g}$ PD-1-lalL-2 or PD-1-wtIL-2 on day 17. Tumor growth was assessed twice a week. (D) A20 tumor-bearing mice ( $n=5$ /group) were treated with equal molar amounts of Erb-lalL-2 (20 $\mu$ g), anti-PD-L1 (10 $\mu$ g), or PD-L1-lalL-2 (20 $\mu$ g) on day 14. Tumor growth was assessed twice a week. (E) A20 tumor-bearing mice ( $n=5$ /group) were treated with $20 \mu g$ Erb-lalL-2, PD-L1-lalL-2, or PD-1-lalL-2 on day 14. Tumor growth was assessed twice a week. (F) A20 tumor-bearing mice ( $n=5$ /group) were treated with $20 \mu g$ PD-1-lalL-2 and/or $100 \mu g$ anti-PD-L1 on day 20. Tumor growth was assessed twice a week. (G) A375 tumor-bearing humanized mice ( $n=5 /$ group) were treated with equal molar amounts of Erb-lalL-2 $(20 \mu \mathrm{g})$ and anti-hPD-1 $(10 \mu \mathrm{g})$ or hPD-1-lalL-2 $(20 \mu \mathrm{g})$ on day 11. Tumor growth was assessed twice a week. Data represent mean \pm SEM from 2 to 3 independent experiments. The $P$ value was determined by 2 -way ANOVA with Geisser-Greenhouse correction $(\mathbf{A}-\mathbf{G}){ }^{* *} P<0.01$ and ${ }^{* * * *} P<0.0001$. 


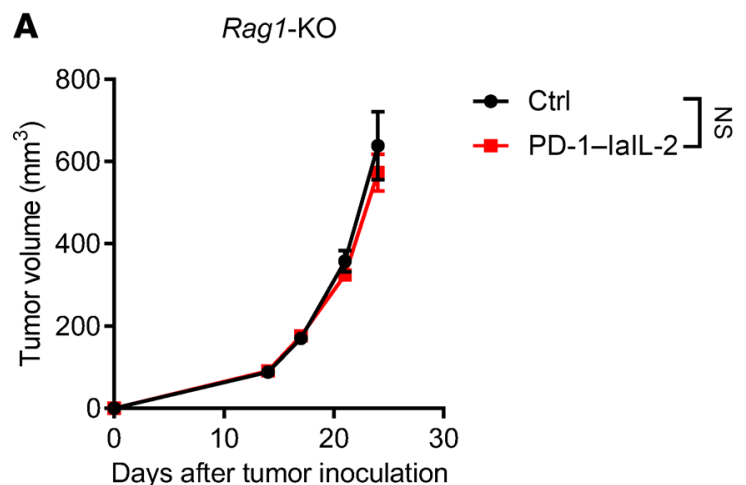

C

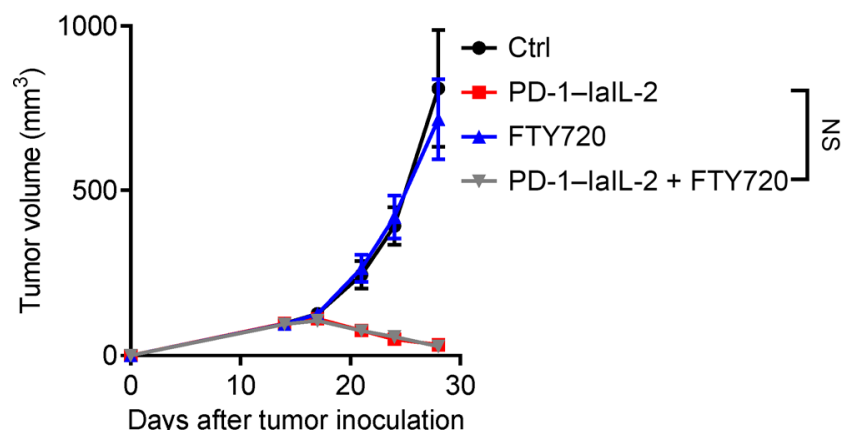

Days after tumor inoculation

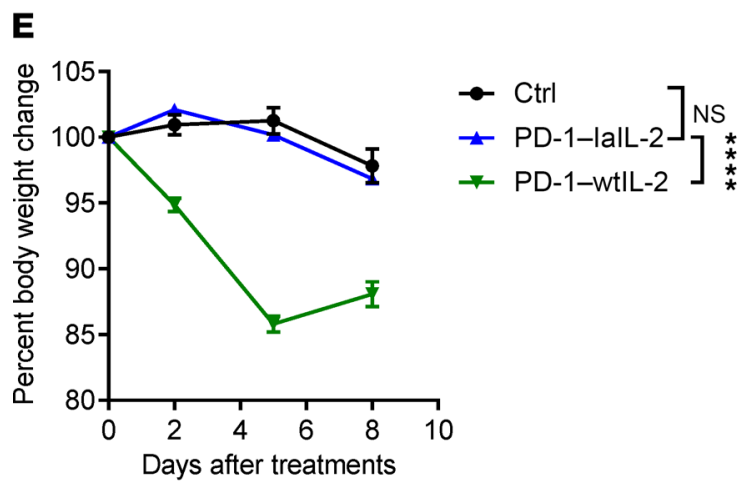

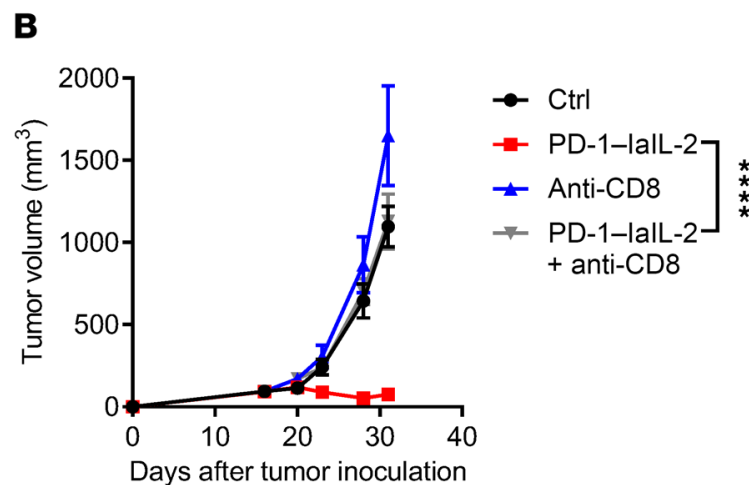
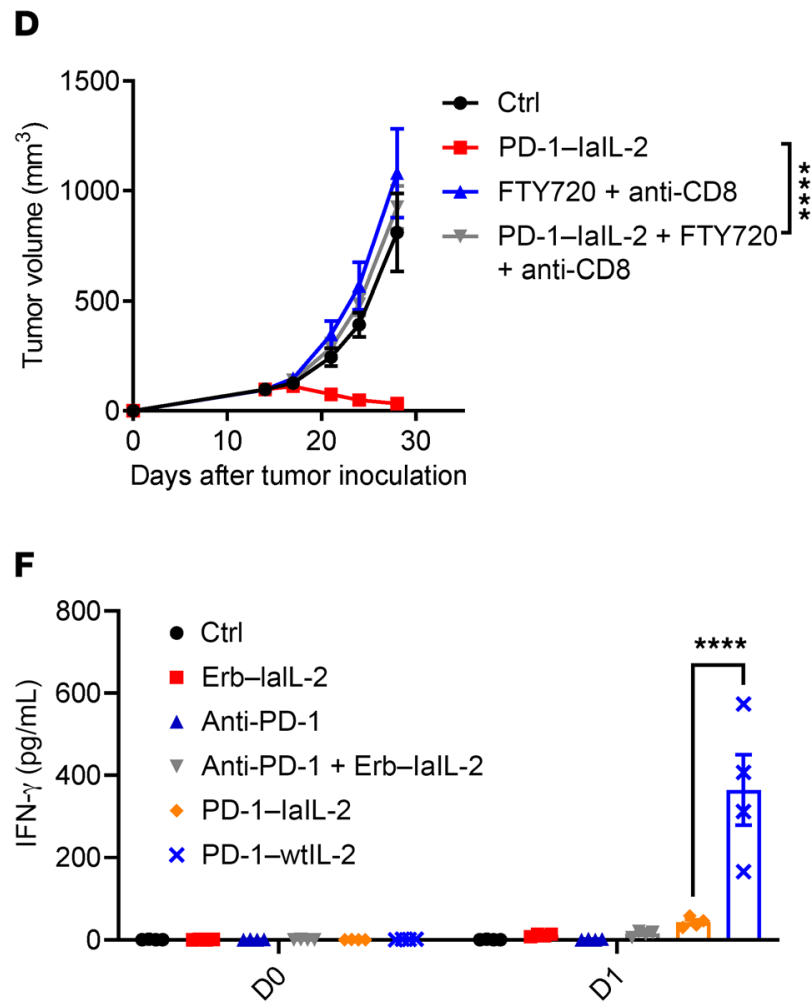

Figure 3. Antitumor efficacy of PD-1-lalL-2 depends on intratumoral CD8 ${ }^{+}$T cells. (A) A20 tumor-bearing Rag1-KO mice $(n=5 / g r o u p)$ were treated with $20 \mu \mathrm{g}$ PD-1-lalL-2 on day 15. Tumor growth was assessed twice a week. (B) A20 tumor-bearing mice ( $n=5 / g r o u p)$ were treated with $20 \mu \mathrm{g}$ PD-1-lalL-2 on day 17. Anti-CD8 (200 $\mu \mathrm{g} /$ mouse) was administered twice a week starting on day 16. Tumor growth was assessed twice a week. (C) A20 tumor-bearing mice ( $n=5$ /group) were treated with $20 \mu \mathrm{g}$ PD-1-lalL-2 on day 17 . FTY720 was administered every 2 days starting on day 16 through the end of the experiment. Tumor growth was assessed twice a week. (D) A20 tumor-bearing mice ( $n=5 /$ group) were treated with $20 \mu \mathrm{g}$ PD-1-lalL-2 on day 17. FTY720 was administered every 2 days starting on day 16 through the end of the experiment. Anti-CD8 (200 $\mu \mathrm{g} /$ mouse) was administered twice a week starting on day 16. Tumor growth was assessed twice a week. (E) Renca tumor-bearing mice ( $n=5$ /group) were treated with $100 \mu$ g PD-1-lalL-2 or PD-1-wtIL-2 intraperitoneally. Mouse body weight was monitored twice a week. (F) Renca tumor-bearing mice ( $n=5 / g r o u p)$ were treated with equal molar amounts of Erb-lalL-2 $(100 \mu \mathrm{g})$, anti-PD-1 $(50 \mu \mathrm{g})$, PD-1-lalL-2 $(100 \mu \mathrm{g})$, or PD-1-wtIL-2 $(100 \mu \mathrm{g})$ by intraperitoneal injection. IFN- $\gamma$ in the serum was measured by cytometric bead array (CBA) one day after the treatment. Data represent mean \pm SEM from 2 independent experiments. The $P$ value was determined by 2 -way ANOVA with Geisser-Greenhouse correction (A-E) or 2-way ANOVA with Tukey's multiple comparisons test (F). ${ }^{* * *} P<0.0001$.

Whether $\mathrm{T}$ cells inside the tumor or draining lymph nodes (dLNs) play a more dominant role in anti-PD-1 therapy remains controversial $(40,41)$. To investigate whether the $\mathrm{T}$ cells in the tumor or dLNs are essential for PD-1-laIL-2 treatment in advanced tumors, we used FTY720 to block sphingosine 1-phosphate receptor 1, thereby prohibiting $\mathrm{T}$ cells from exiting the lymphoid organs (42). Intriguingly, FTY720 treatment had no impact on the therapeutic effect of PD-1-laIL-2 (Figure 3C), which suggests that
T cells from dLNs are not necessary for PD-1-laIL-2 treatmentinduced tumor control. Further depletion of $\mathrm{CD} 8^{+} \mathrm{T}$ cells during FTY720 treatment confirmed that $\mathrm{CD} 8^{+} \mathrm{T}$ cells inside the tumor were necessary for PD-1-laIL-2-induced tumor control (Figure 3D). Therefore, for established tumors, TILs might be essential for tumor control after the systemic delivery of fusion proteins.

In the clinic, high-dose IL-2 treatment causes severe side effects $(30,43)$. To test whether PD-1-laIL-2 treatment also induces side 

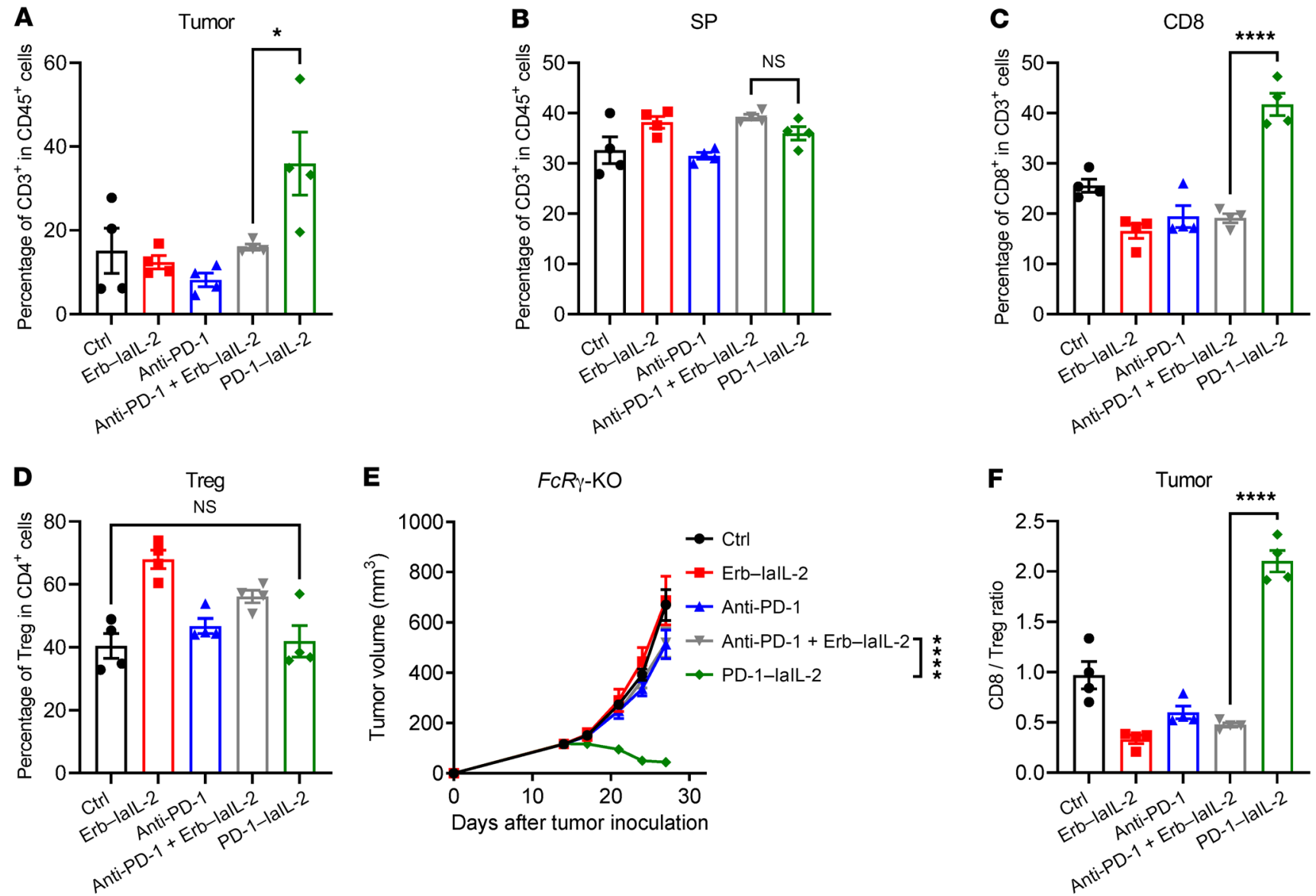

G

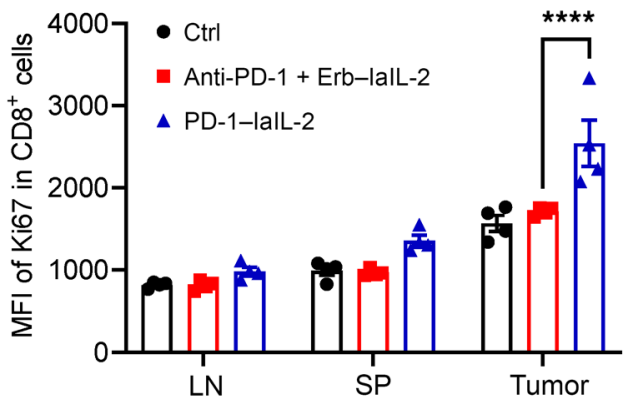

H
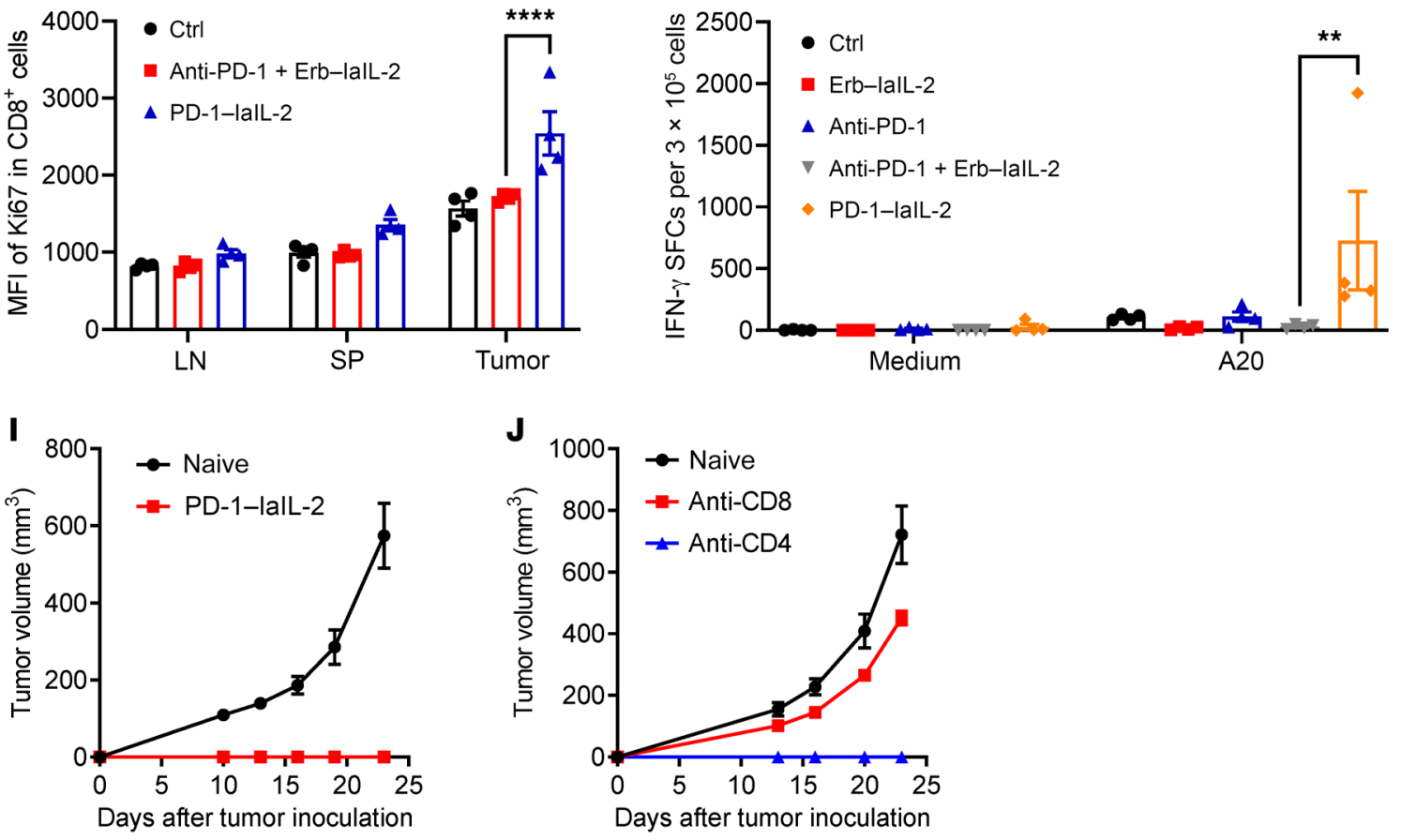
Figure 4. PD-1-lalL-2 increases the abundance of tumor-specific CD8 T cells. (A-D) A20 tumor-bearing mice ( $n=5$ /group) were treated with equal molar amounts of Erb-lalL-2 $(20 \mu \mathrm{g})$, anti-PD-1 (10 $\mu \mathrm{g})$ or PD-1-lalL-2 $(20 \mu \mathrm{g})$ on day 17. Six days later, T cells from the tumor and spleen were analyzed. CD3 ${ }^{+} \mathrm{T}$ cell frequency in the tumor or spleen from different groups is shown separately in $\mathbf{A}$ and $\mathbf{B}$. CD8 ${ }^{+} \mathrm{T}$ cell or Treg cell frequencies in tumors from different groups are shown separately in $\mathbf{C}$ and $\mathbf{D}$. (E) A20 tumor-bearing $F c R \gamma$ KO mice ( $n=5 /$ group) were treated with equal molar amounts of Erb-lalL-2 $(20 \mu \mathrm{g})$, anti-PD-1 $(10 \mu \mathrm{g})$, or PD-1-lalL-2 $(20 \mu \mathrm{g})$ on day 15. Tumor growth was assessed twice a week. (F and $\mathbf{C})$ The mice were treated as in A-D. Six days later, T cells from the tumor and spleen were analyzed. The ratio of $\mathrm{CD} 8^{+} T$ cells to Treg cells is shown in $\mathbf{F}$. Ki67 expression in CD8 ${ }^{+}$T cells is shown in $\mathbf{G}$. (H) The mice were treated as in $\mathbf{A}-\mathbf{F}$. Six days later, draining lymph node (dLN) cells were collected, and the IFN- $\gamma$ ELISPOT assay was performed with medium or irradiated A20 cell restimulation. (I and J) Approximately 60 days after tumor rejection in PD-1-lalL-2treated mice ( $n=10 /$ group), 5 times the original number of A20 cells $\left(1 \times 10^{7}\right)$ were injected on the opposite flank for tumor rechallenge. AntiCD8 (200 $\mu \mathrm{g} /$ mouse) or anti-CD4 (200 $\mu \mathrm{g} /$ mouse) was administered twice a week starting 1 day before tumor inoculation. Naive WT BALB/c mice ( $n=5$ /group) were used as controls. Tumor growth was monitored twice a week. Data represent mean \pm SEM from 2 independent experiments. The $P$ value was determined by 1-way ANOVA with Tukey's multiple comparisons test (A-D and F), 2-way ANOVA with Geisser-Greenhouse correction (E), or 2-way ANOVA with Tukey's multiple comparisons test (G and $\mathbf{H}) .{ }^{*} P<0.05$, ${ }^{* *} P<0.01$, and ${ }^{* * *} P<0.0001$.

effects, we monitored the body weights of mice during and after the treatment. The body weights of mice were reduced only in the PD-1-wtIL-2-treated group but not in the PD-1-laIL-2-treated group (Figure 3E), which suggests that PD-1-laIL-2 has much lower toxicity than PD-1-wtIL-2. Additionally, there was a much lower level of IFN- $\gamma$ in the serum of PD-1-laIL-2-treated mice than in that of PD-1wtIL-2-treated mice (Figure 3F). Together, these data suggest that PD-1-laIL-2 is much safer and more effective than PD-1-wtIL-2.

PD-1-laIL-2 increases the abundance of tumor-specific $C D 8^{+} T$ cells. As T cells are important for PD-1-laIL-2 treatment and PD-1laIL-2 does not bind to peripheral T cells, we proposed that PD-1laIL-2 can preferentially expand TILs but not $\mathrm{T}$ cells in lymphoid tissues. Indeed, the abundance of $\mathrm{T}$ cells in the tumor increased after PD-1-laIL-2 treatment but showed no changes in the combination-treated group (Figure 4A). Interestingly, the $\mathrm{T}$ cells in the spleen and dLNs did not increase after PD-1-laIL-2 treatment (Figure 4B and Supplemental Figure 4A). Similarly, PD-1-laIL-2 induced an increase in $\mathrm{CD}^{+}$TILs but not $\mathrm{CD}^{+} \mathrm{T}$ cells in the dLNs or spleen (Figure 4C and Supplemental Figure 4, B and C). This result is consistent with our previous finding that PD-1-laIL-2 could not bind to peripheral T cells (Figure 1I). Treg cells suppress the proliferation and expansion of $\mathrm{CD}^{+} \mathrm{T}$ cells, so PD-1-laIL-2 may indirectly induce an increase in $\mathrm{CD}^{+} \mathrm{T}$ cells through Treg cell depletion. However, the percentage of Treg cells did not change in the tumor (Figure 4D) but increased in the dLNs and spleen (Supplemental Figure 4, D and E). This might suggest that PD-1laIL-2 does not induce Treg cell depletion. Since the depletion of Tregs by antibodies depends on the $\gamma$ subunit of the immunoglobulin Fc receptor ( $\mathrm{FcR} \gamma$ ), we utilized $F c R \gamma$ knockout (KO) mice to study whether this fusion protein depends on $\mathrm{FcR} \gamma$ for its activity. PD-1-laIL-2 could efficiently control the tumors in FcR $\gamma$-KO mice (Figure 4E), which further proves that the therapeutic function of PD-1-laIL-2 does not depend on Treg depletion. There was a 4-fold increase in the $\mathrm{CD} 8^{+} \mathrm{T}$ cell to Treg cell ratio in the tumor after PD-1laIL-2 treatment (Figure 4F), suggesting that PD-1-laIL-2 promotes an immune-active environment in the tumor. Moreover, the ratio of $\mathrm{CD}^{+} \mathrm{T}$ cells to Treg cells decreased in the dLNs and spleen (Supplemental Figure 4, F and G), which is consistent with the lack of toxicity observed in PD-1-laIL-2-treated mice. To investigate how the $\mathrm{CD}^{+} \mathrm{T}$ cell number increases, we examined Ki67 expression in $\mathrm{CD}^{+} \mathrm{T}$ cells. After PD-1-laIL-2 treatment, $\mathrm{CD} 8^{+} \mathrm{T}$ cells expressed much higher Ki67 levels (Figure 4G), which suggests that PD-1laIL-2 promotes the proliferation of $\mathrm{CD}^{+} \mathrm{T}$ cells in the tumor.

To determine whether this fusion protein directly activates and expands tumor-specific $\mathrm{T}$ cells, we isolated immune cells from the dLNs and restimulated them with irradiated A20 tumor cells. The IFN- $\gamma$ ELISPOT assay showed many more immunospots after PD-1-laIL-2 treatment (Figure 4H), which indicates that PD-1-laIL-2 directly increases the tumor-specific T cell response. To test whether PD-1-laIL-2 treatment induces a protective antitumor memory response, we injected 5 times the original number of tumor cells into the opposite site of the treated back of the PD-1laIL-2-treated mice at approximately 2 months after eliminating the tumors from those mice. All cured mice spontaneously rejected the rechallenged tumors (Figure 4I). However, if the CD8 ${ }^{+} \mathrm{T}$ cells were depleted before rechallenging, all the mice developed tumors (Figure 4J), indicating the important role of $\mathrm{CD}^{+} \mathrm{T}$ cells in protection. $\mathrm{CD} 4^{+} \mathrm{T}$ cells were dispensable for protection (Figure $4 \mathrm{~J})$. These data suggest that PD-1-laIL-2 increases the abundance of tumor-specific $\mathrm{T}$ cells and induces a potent memory response.

$P D-1-$ laIL-2 causes the proliferation of $P D-1^{+} T I M 3^{+} C D 8^{+}$effector $T$ cells. To investigate the functional changes in TILs at the single-cell level after PD-1-laIL-2 treatments, we isolated T cells from the tumor for single-cell RNA sequencing. The T cells could be divided into 16 clusters with unsupervised clustering (Supplemental Figure 5A). According to CD8b1 expression, there were 4 $\mathrm{CD}^{+} \mathrm{T}$ cell clusters (clusters 2, 4, 5, and 7; Figure 5A), and PD-1laIL-2 specifically increased the abundance of cluster 5 of $\mathrm{CD}^{+} \mathrm{T}$ cells (Figure 5B and Supplemental Figure 5B). Next, we examined the proteins expressed in cluster 5 . As expected, cluster 5 had high expression of Gzma (Figure 5C) and IFN- $\gamma$ (Figure 5D). To our surprise, similar to cluster 2, cluster 5 also expressed high levels of PD-1 (Figure 5E) and TIM3 (Figure 5F). However, cluster 5 also expressed a high level of Ki67 (Figure 5G), making it different from cluster 2 . Since $\mathrm{PD}-1^{+} \mathrm{TIM}^{+} \mathrm{T}$ cells are often considered to be dysfunctional and terminally differentiated TILs, our data raise the possibility that PD-1-laIL-2 can promote the proliferation of $\mathrm{PD}-1^{+} \mathrm{TIM}^{+} \mathrm{CD} 8^{+} \mathrm{T}$ cells with effector function.

PD-1-laIL-2 promotes TIL differentiation into proliferating $P D-1^{+} \mathrm{TIM}^{+} \mathrm{CD} 8^{+}$effector $\mathrm{T}$ cells. To test whether PD-1-laIL-2 can promote TIL differentiation into proliferating $\mathrm{PD}-1^{+} \mathrm{TIM} 3^{+} \mathrm{CD} 8^{+}$ $\mathrm{T}$ cells with effector function, we further analyzed the relationship of $\mathrm{CD}^{+} \mathrm{T}$ cell clusters with Monocle. Indeed, $\mathrm{T}$ cells in cluster 5 and cluster 2 were all terminally differentiated TILs with distinct phenotypes (Figure 6A and Supplemental Figure 5C), which suggests that PD-1-laIL-2 can promote $\mathrm{CD}^{+} \mathrm{T}$ cells to differentiate into proliferating effector TILs. Pathway analysis showed the enrichment of IL-2/STAT5 signaling pathway-related genes (Figure $6 \mathrm{~B}$ ), $\mathrm{T}$ cell receptor signaling pathway-related genes (Figure $6 \mathrm{C}$ ), and IFN- $\gamma$ response-related genes (Figure 6D) in cluster 
A

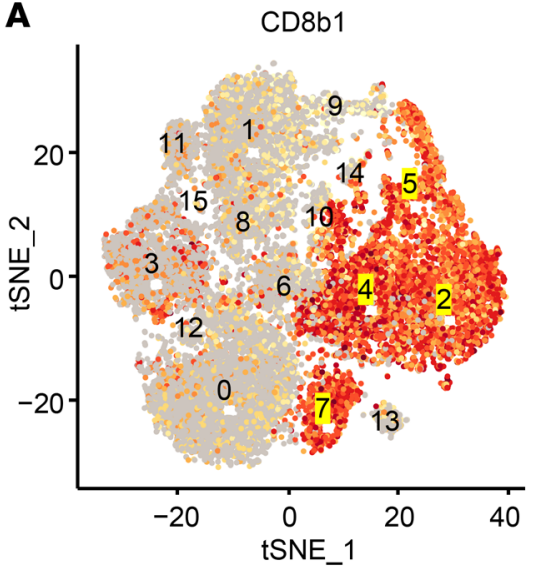

C

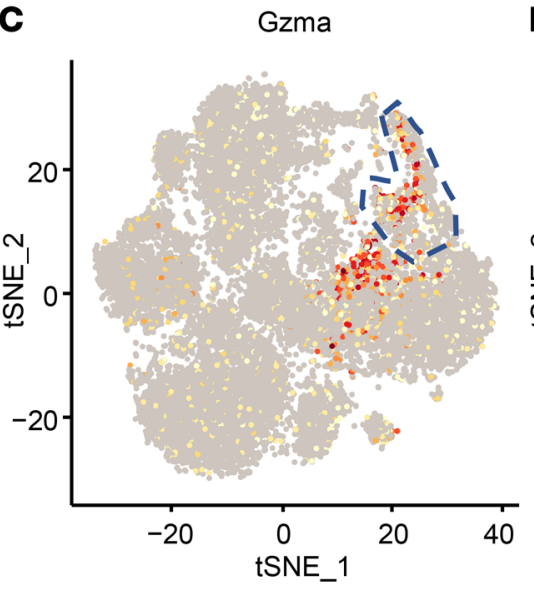

$\mathbf{F}$

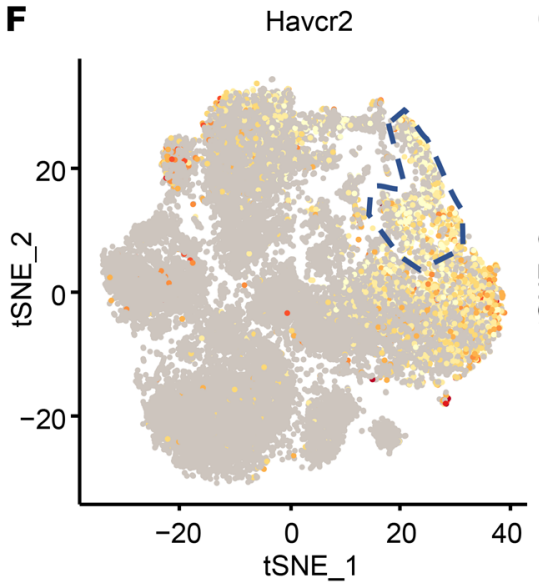

B Ctrl

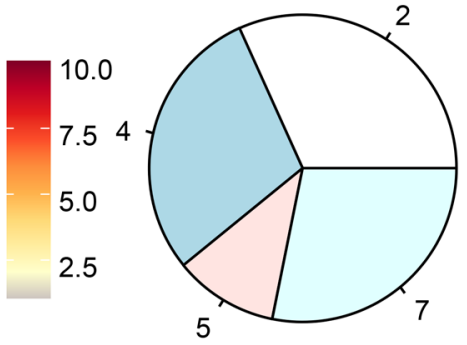

D

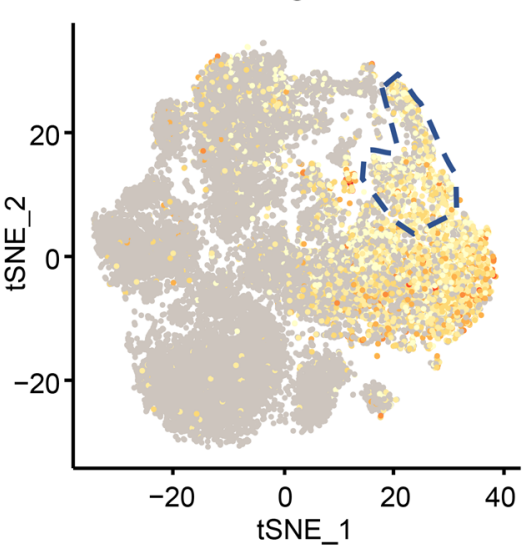

G

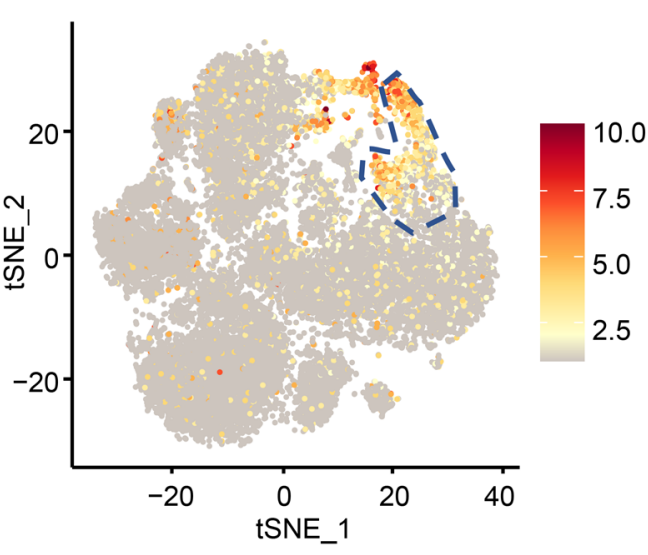

10.0

7.5

5.0

.5
PD-1_Erb.lalL-2

PD-1.lalL-2
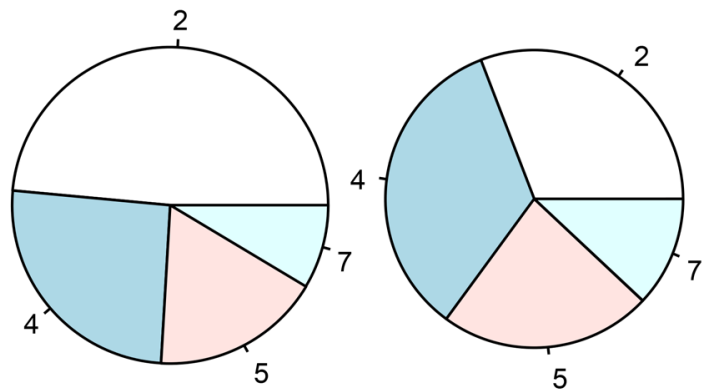

E Pdcd1

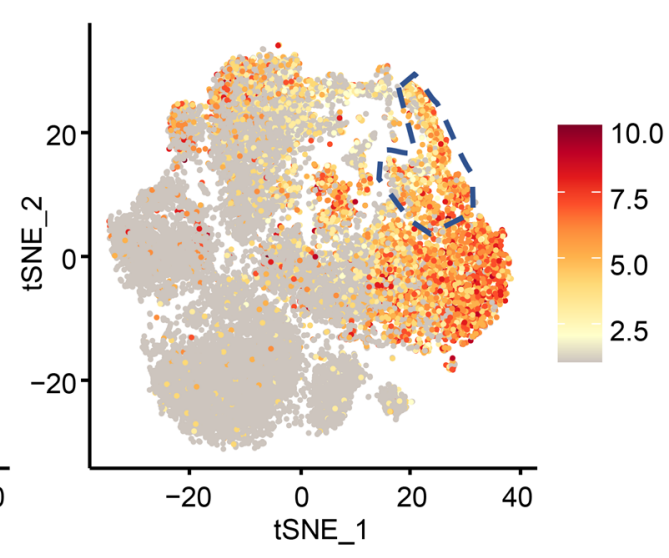

Figure 5. PD-1-lalL-2 causes the proliferation of PD-1+TIM3+CD8+ effector T cells. A20 tumor-bearing mice ( $n=5 / g r o u p)$ were treated with equal molar amounts of Erb-lalL-2 $(20 \mu \mathrm{g})$ and anti-PD-1 $(10 \mu \mathrm{g})$ (PD-1_Erb.lalL-2) or PD-1-lalL-2 $(20 \mu \mathrm{g})$ on day 17. Three days later, CD3 ${ }^{+} \mathrm{T}$ cells from the tumor were sorted for single-cell RNA sequencing. (A) Cd8b1 expression in each cluster. (B) Percentages of each cluster in CD8 ${ }^{+}$T cell clusters. (C-C) Gzma (C), IFN- $\gamma$ (D), PD-1 (E), TIM3 (F), and Ki67 (C) expression in each cluster. Cluster 5 was labeled.

5 , which indicates that $\mathrm{T}$ cells in cluster 5 are enriched tumorspecific $\mathrm{T}$ cells and respond more readily to IL-2 stimulation. Taken together, these findings indicate that PD-1-laIL-2 increases the abundance of proliferating PD-1+TIM3 $3^{+} \mathrm{CD} 8^{+}$effector T cells.

$P D$-1-lalL-2 specifically reactivates $P D-1^{+} T I M 3^{+}$tumor-specific $C D 8^{+} T$ cells. To confirm whether PD-1-laIL-2 can increase the effector function of some subsets of TILs, we sorted CD $4^{+}, \mathrm{PD}-1^{-} \mathrm{CD} 8^{+}$,
PD- $1^{+} \mathrm{TIM}^{-} \mathrm{CD}^{+}$, and $\mathrm{PD}-1^{+} \mathrm{TIM}^{+} \mathrm{CD}^{+} \mathrm{T}$ cells from the tumor and treated them with PD-1-laIL-2 in the presence of irradiated A20 tumor cells (Figure 7A). It is widely believed that PD-1- TILs might be newly arrived cells, PD-1+TIM3- TILs are newly activated effector cells and PD-1 $1^{+} \mathrm{TIM}^{+}$TILs are exhausted cells $(44,45)$. To our surprise, PD-1-laIL-2 could not induce IFN- $\gamma$ expression in $\mathrm{CD}^{+}, \mathrm{PD}^{-{ }^{-} \mathrm{CD}} 8^{+}$or $\mathrm{PD}-1^{+} \mathrm{TIM} 3^{-} \mathrm{CD} 8^{+} \mathrm{T}$ cells (Figure $7, \mathrm{~B}$ and 


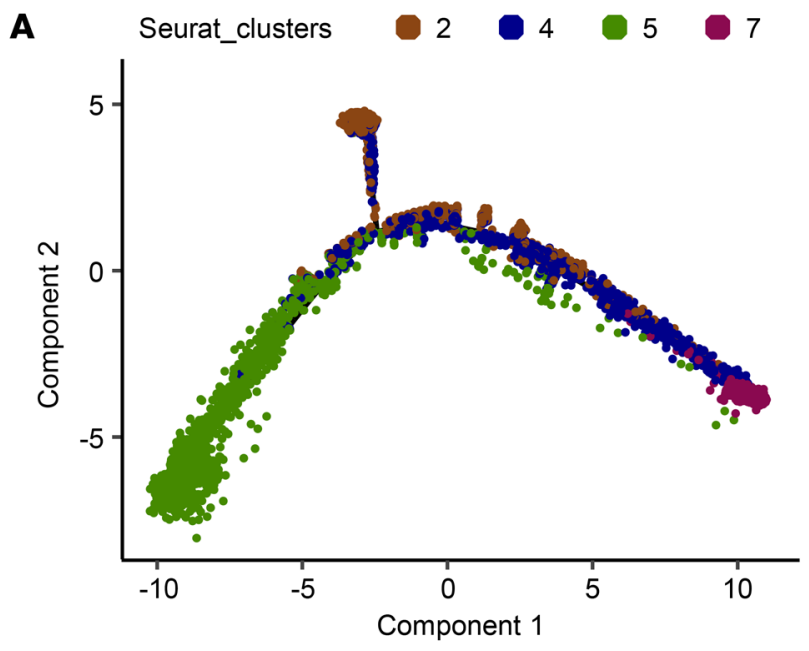

C

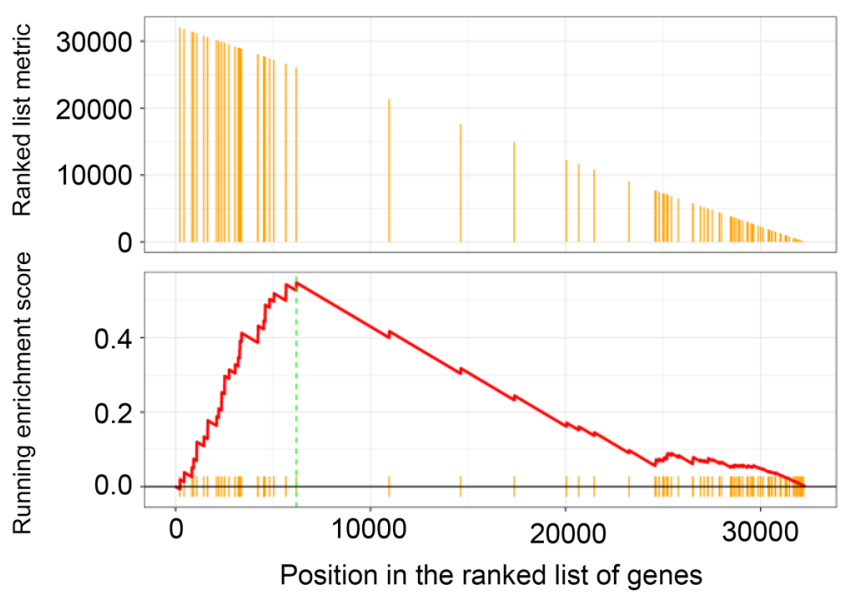

B

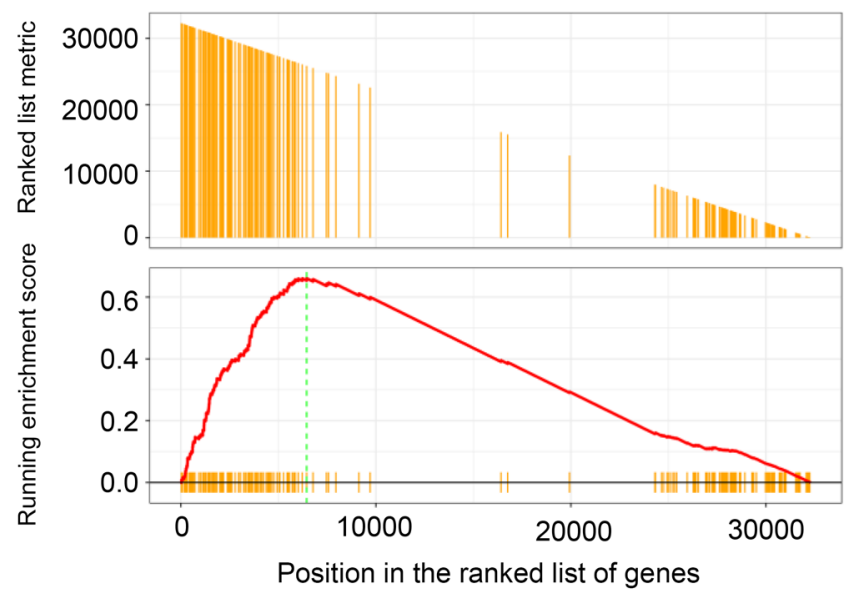

D

IFN- $\gamma$ response

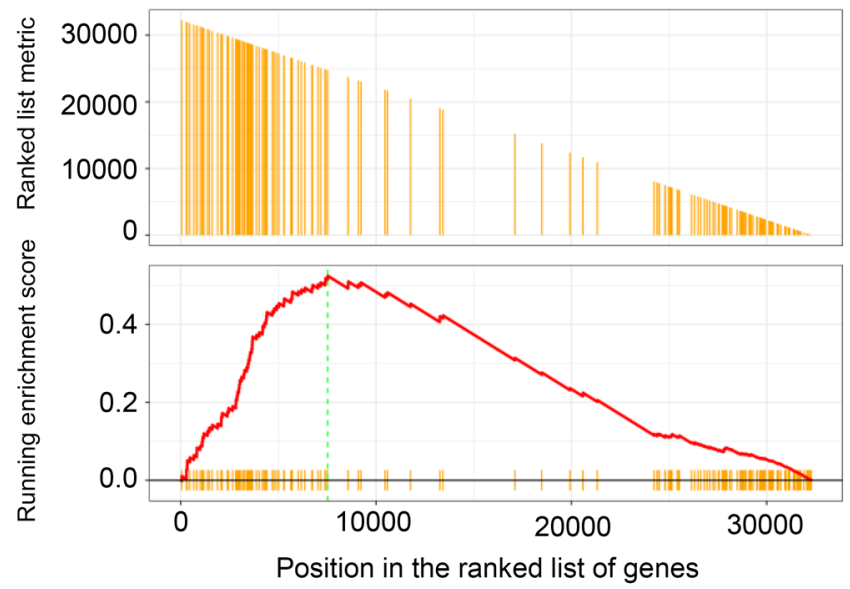

Figure 6. PD-1-lalL-2 promotes TIL differentiation into proliferating PD-1+TIM3+CD8 ${ }^{+}$effector $\mathbf{T}$ cells. A2O tumor-bearing mice $(n=5 / g r o u p)$ were treated with equal molar amounts of Erb-lalL-2 $(20 \mu \mathrm{g})$ and anti-PD-1 $(10 \mu \mathrm{g})(\mathrm{PD}-1$-Erb.lalL-2) or PD-1-lalL-2 (20 $\mu \mathrm{g})$ on day 17 . Three days later, CD3 ${ }^{+} \mathrm{T}_{\text {cells from }}$ the tumor were sorted for single-cell RNA sequencing. (A) Single-cell trajectories of CD8 ${ }^{+}$T cell clusters. (B-D) Gene set enrichment analysis of IL-2-STAT5 signaling (B), T cell receptor signaling (C), and IFN- $\gamma$ (D) response-related genes in cluster 5 compared with other CD8 $8^{+}$T cell clusters.

C, and Supplemental Figure 6A). However, IFN- $\gamma$-producing cells stimulated by tumors were primarily enriched inside the population of PD- ${ }^{+} \mathrm{TIM}^{+}{ }^{+} \mathrm{CD} 8^{+} \mathrm{T}$ cells after PD-1-laIL-2 treatment (Figure 7D). These data suggest that PD-1-laIL-2 could increase the function of $\mathrm{PD}-1^{+} \mathrm{TIM}^{+} \mathrm{CD} 8^{+} \mathrm{T}$ cells. Furthermore, these results also indicate that $\mathrm{PD}-1^{+} \mathrm{TIM}^{+}$cells are the tumor-specific $\mathrm{T}$ cells in the tumor. To directly check the expression of PD-1 and TIM3 on tumor-specific T cells, we used tetramers to track tumor-specific T cells in the MC38 tumor model. The results showed that most of the tetramer-positive CD8 ${ }^{+} \mathrm{T}$ cells were PD- 1 and TIM3 double positive (Figure 7E and Supplemental Figure 6B). Therefore, most tumor-specific $\mathrm{T}$ cells responding to IL- 2 are $\mathrm{PD}-1^{+} \mathrm{TIM} 3^{+}$cells.

To investigate whether PD-1-laIL-2 can directly promote the proliferation of $\mathrm{PD}-1^{+} \mathrm{TIM} 3^{+} \mathrm{CD} 8^{+} \mathrm{T}$ cells, we labeled PD $-1^{+} \mathrm{TIM}^{+}{ }^{+} \mathrm{CD}^{+}{ }^{+} \mathrm{T}$ cells isolated from anti-CD3- and anti-CD28treated splenocytes with carboxyfluorescein succinimidyl ester (CFSE) and treated them with PD-1-laIL-2 or the combination of anti-PD-1 and Erb-laIL-2. Two days later, we examined the morphological phenotypes and CFSE density of these T cells with an
Incucyte system. PD-1-laIL-2 greatly increased the size of the $\mathrm{T}$ cell clusters (Figure $7 \mathrm{~F}$ and Supplemental Figure 6C), which suggests that PD-1-laIL-2 increases the total number of $\mathrm{T}$ cells. PD-1-laIL-2 also reduced the mean green intensity (Figure $7 \mathrm{G}$ and Supplemental Figure 6C), indicating the dilution of CFSE after cell division. We also confirmed the reduction in CFSE brightness by flow cytometry (Figure 7, $\mathrm{H}$ and I, and Supplemental Figure 6D). To examine whether PD-1-laIL-2 can effectively recover the functionality of dysfunctional $\mathrm{T}$ cells, we examined the cytotoxic function of $\mathrm{PD}-1^{+} \mathrm{TIM}^{+} \mathrm{CD} 8^{+} \mathrm{T}$ cells from the tumor-bearing mice after PD-1-laIL-2 treatment. Indeed, PD-1-laIL-2 increased the frequency of CD107a $\mathrm{a}^{+} \mathrm{IFN}-\gamma^{+}$cells among PD $-1^{+} \mathrm{TIM} 3^{+} \mathrm{CD} 8^{+} \mathrm{T}$ cells in the TME (Figure 7J). Therefore, PD-1-laIL-2 can reactivate $\mathrm{PD}-1^{+} \mathrm{TIM}^{+}$tumor-specific $\mathrm{CD} 8^{+} \mathrm{T}$ cells in the tumor.

\section{Discussion}

Immunogenic tumor tissues often have a high number of $\mathrm{T}$ cells, but their dysfunction limits their capacity to control tumors (13, 46). $\mathrm{PD}-1^{+}$and $\mathrm{TIM} 3^{+}$TILs are considered to be terminally differ- 
A

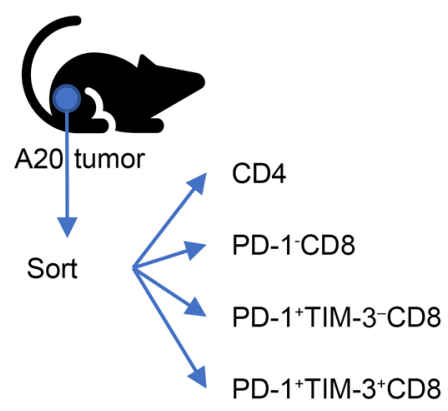

C $\quad \mathrm{PD}-1^{+} \mathrm{TIM}-3-\mathrm{CD} 8$

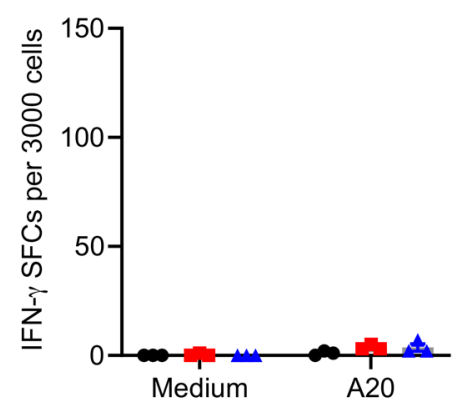

E
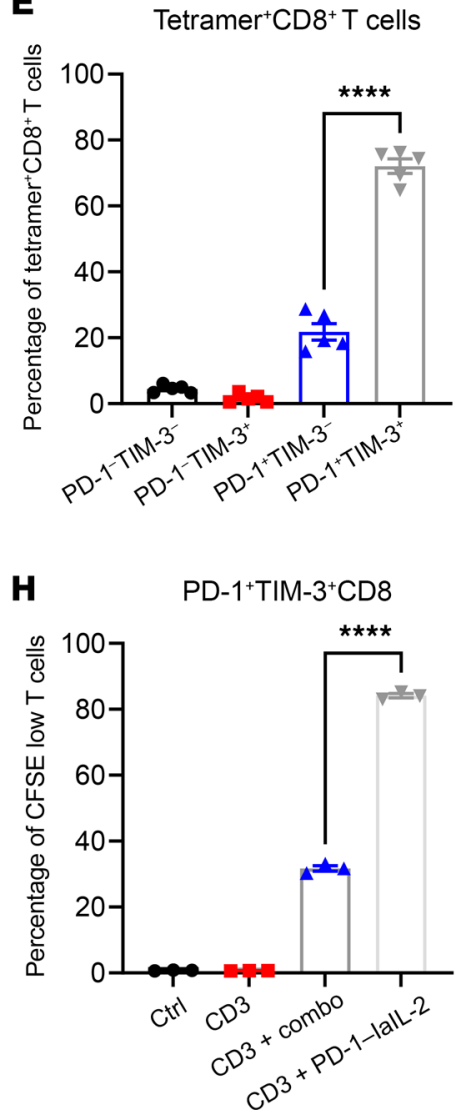

B

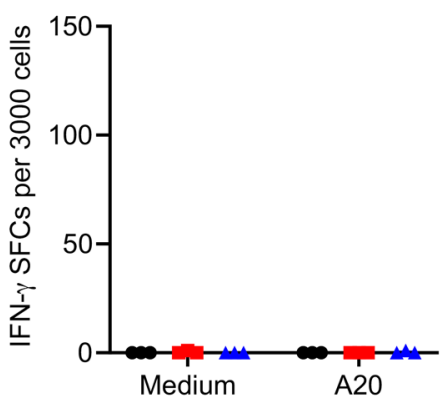

D $\quad$ PD-1+TIM-3+CD8

- Medium

- Erb-lalL-2

A PD-1-lalL-2
- Medium

- Erb-lalL-2

A PD-1-lalL-2

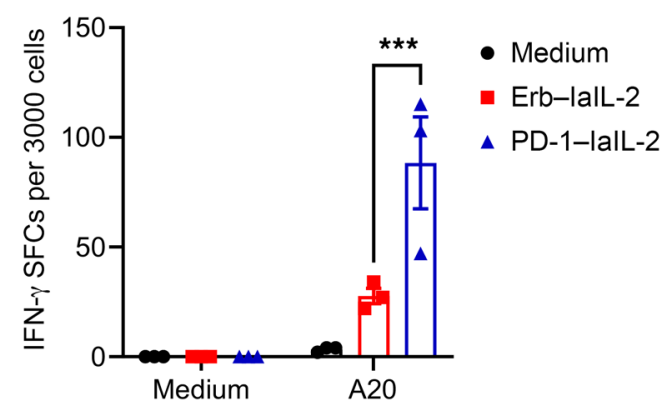

$\mathbf{F}$

PD $-1^{+}$TIM- $3^{+}$CD 8

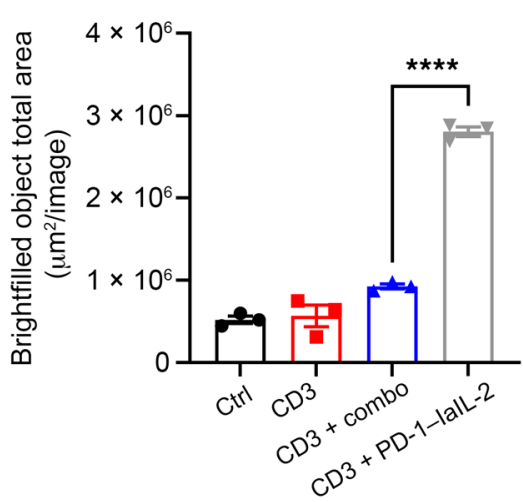

I PD-1+TIM- $3^{+} \mathrm{CD} 8$

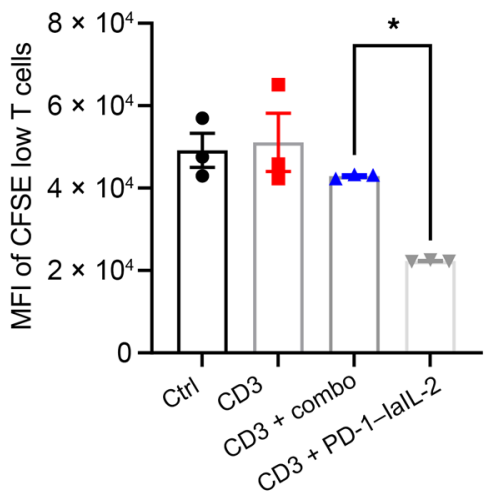

G

PD $-1+$ TIM $-3^{+}$CD8
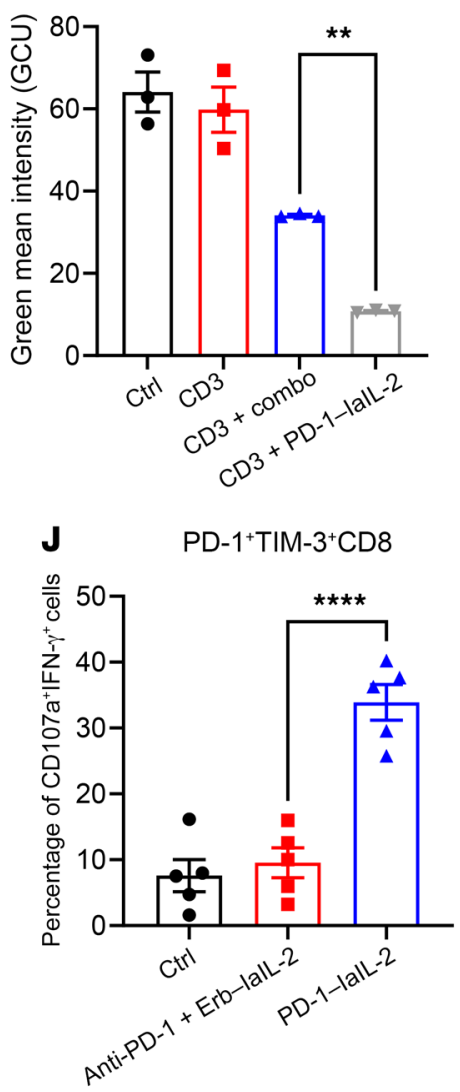
Figure 7. PD-1-lalL-2 specifically reactivates $\mathrm{PD}-1^{+} \mathrm{TIM3}^{+}$tumor-specific CD8 ${ }^{+}$T cells. (A-D) CD4 ${ }^{+}, \mathrm{PD}-1^{-} \mathrm{CD}^{+}, \mathrm{PD}-1^{+} \mathrm{TIM} 3^{-} \mathrm{CD}^{+}$, and $\mathrm{PD}-1^{+} \mathrm{TIM} 3^{+} \mathrm{CD} 8^{+}$ $T$ cells from $A 20$ tumor-bearing mice were sorted out and cocultured with irradiated A20 cells in the presence of Erb-lalL-2 or PD-1-lalL-2 for the IFN- $\gamma$ ELISPOT assay. Experimental scheme (A) and spots from PD-1-CD8 (B), PD-1+TIM3-CD8 ${ }^{+}$(C) and PD-1+TIM3+CD8+ (D) T cells are shown. (E) PD-1 and TIM3 expressions on tetramer+CD8 ${ }^{+} T$ cells in tumors from MC38 tumor-bearing mice $(n=5)$. ( $F-I)$ Splenocytes were stimulated with antiCD3 and anti-CD28 antibodies. Five days later, PD- $1^{+}{ }^{T} I M 3^{+} C D 8^{+} T$ cells were sorted out and labeled with CFSE. Then, the cells were cultured in 96-well plates in the presence of anti-CD3, Erb-lalL-2 plus anti-PD-1 (combo), or PD-1-lalL-2 for 2 days. The T cell clusters and CFSE expression were assayed with an Incucyte instrument. Total areas of the cluster are shown in F. Mean CFSE intensity is shown in G. (H) Percentage of CFSE low cells. (I) MFI of CFSE low cells. (J) A20 tumor-bearing mice ( $n=5 /$ group) were treated with equal molar amounts of Erb-lalL-2 $(20 \mu \mathrm{g})$, anti-PD-1 $(10 \mu \mathrm{g})$, or PD-1-lalL-2 (20 $\mu \mathrm{g})$ on day 19. Six days later, T cells from the tumor were analyzed. Data represent mean \pm SEM. The $P$ value was determined by 2-way ANOVA with Tukey's multiple comparisons test (D) or 1-way ANOVA with Tukey's multiple comparisons test $(\mathbf{E}-\mathrm{J}) .{ }^{*} P<0.05$, ${ }^{* *} P<0.01$, ${ }^{* * *} P<$ 0.001 , and ${ }^{* * * *} P<0.0001$.

entiated and dysfunctional TILs (44). Anti-PD-1/PD-L1 treatment can release the brake on the $\mathrm{T}$ cell response and partially restore their functions, but only a small number of patients have complete responses (9). Unexpectedly, we observed that IL-2 is required for optimal PD-1 therapy. This raises the possibility that targeting TILs with IL-2 might overcome PD-1 resistance. However, due to the high expression of IL-2 receptors on Treg cells, it is difficult to deliver enough $\mathrm{IL}-2$ to $\mathrm{CD} 8^{+} \mathrm{T}$ cells in the tumor while avoiding systemic toxicity. Therefore, we designed a PD-1-laIL-2 fusion protein by linking low-affinity IL-2 to an anti-PD-1 antibody to target intratumoral PD-1-high $\mathrm{CD}^{+} \mathrm{T}$ cells instead of Tregs. PD-1laIL-2 had much lower binding to peripheral $\mathrm{CD} 8^{+} \mathrm{T}$ cells and Treg cells, which greatly reduced peripheral consumption. A single lowdose of PD-1-laIL-2 treatment eradicated the tumors. PD-1-laIL-2 treatment favors $\mathrm{CD} 8^{+} \mathrm{T}$ cells over Treg cells in the tumor. Intriguingly, PD-1-laIL-2 could reactivate PD- $1^{+} \mathrm{TIM}^{+} \mathrm{CD} 8^{+}$TILs, and a long-term memory response was generated to protect against relapse. Overall, PD-1-laIL-2 is a next-generation PD-1 therapy that can target tumor-specific $\mathrm{T}$ cells.

One major obstacle for IL-2 cancer immunotherapy is the high expression of IL-2R $\alpha$ on Treg cells $(30,37)$. Therefore, high-dose IL-2 must be used to increase the accessibility of $\mathrm{CD} 8^{+} \mathrm{T}$ cells to IL-2 in the clinic, which in turn increases severe toxicities due to strong $\mathrm{CD} 8^{+} \mathrm{T}$ cell activation (39). To make IL-2 selectively bind to $\mathrm{CD} 8^{+} \mathrm{T}$ cells, our group and others have tried to reduce the binding of IL-2 to Treg cells $(28,35)$. The F42A mutation was used to reduce the binding of IL-2 to IL-2R $\alpha$. While this mutation reduces the binding to Tregs, it still activates circulating $\mathrm{CD} 8^{+} \mathrm{T}$ cells and increases cytokine release in the periphery (28). The laIL-2 we used in this study maintains the low binding feature to IL-2R $\alpha$ and further reduces binding to IL-2R $\beta$ (47). As a result, laIL-2 cannot activate peripheral $\mathrm{CD} 8^{+} \mathrm{T}$ cells or Treg cells but is able to activate $\mathrm{CD} 8^{+} \mathrm{T}$ cells in the tumor when paired with anti-PD-1. This is critical in reducing IL-2 treatment-induced systemic toxicity. During the revision of our manuscript from a preprint website (Research Square), we noticed that Roche is also testing a similar fusion protein. The IL-2 variant that they use is only devoid of IL-2R $\alpha$ binding. This is different from the IL- 2 we use in the present study, as we also reduce the IL- 2 binding to IL-2R $\beta$, which will not induce systemic toxicity.

Most of the studies focus on targeting $\mathrm{T}$ cell-driven cytokines to tumor cells or tumor stromal cells $(32,34,36,37)$. This will increase cytokine retention in tumor tissues, but the accessibility of effector $\mathrm{T}$ cells to those cytokines might still be limited. It is unclear whether tumor cells or stromal cells are the most desirable targets for $\mathrm{T}$ cellassociated cytokines, as some tumor cells are not in contact with $\mathrm{T}$ cells, and the internalization of cytokines by tumor cells might reduce the accessibility of TILs to cytokines. Current studies suggest that targeting cytokines to intratumoral effector $\mathrm{T}$ cells, preferentially CD8 ${ }^{+}$TILs, might be an efficient yet challenging approach to cancer immunotherapy. The ideal design is targeting cytokines to tumor-specific T cells. However, to the best of our knowledge, no unique markers of tumor-specific $\mathrm{T}$ cells have been identified and utilized. An alternative candidate molecule may have the following features: (a) high expression on intratumoral $\mathrm{CD}^{+} \mathrm{T}$ cells, (b) functional involvement in antigen-driven $\mathrm{T}$ cell activation, and (c) a defined cell subtype that is sensitive to cytokine accessibility. Previous studies have shown that PD-1 is highly expressed on antigen-experienced $\mathrm{T}$ cells (48). These cells are the principal subsets responding to immunotherapy and might rely on additional cytokine signaling for rapid antigen-driven proliferation. These features make PD-1 an intriguing target for delivering IL-2 to antigen-specific $\mathrm{T}$ cells in the tumor. Indeed, a previous study suggested that combining PD-L1 blockade with IL-2 administration augments T cell responses under conditions of chronic infection (49). This strategy might not be applied to cancer immunotherapy because tumor microenvironments are heavily infiltrated with immunosuppressive Treg cells that are more likely to benefit from IL-2 $(50,51)$. Our PD-1-laIL-2 specifically targets laIL-2 to antigen-specific intratumoral $\mathrm{CD} 8^{+} \mathrm{T}$ cells and benefits effector $\mathrm{T}$ cells instead of Treg cells.

The insufficiency of IL-2 may be a critical issue in T cell exhaustion development. Interestingly, tumor-specific $\mathrm{T}$ cells are enriched in PD-1 $1^{+} \mathrm{IM} 3^{+} \mathrm{TILs}$, an assumed exhausted $\mathrm{T}$ cell population. Indeed, our PD-1-laIL-2 specifically delivers IL-2 to exhausted tumor-specific $\mathrm{T}$ cells marked by high PD-1 and TIM3 expression and expands them with enhanced antitumor polyfunctionality, as indicated by high expression of IFN- $\gamma$ and CD107a. This implies that IL-2 signaling might potentially antagonize PD-1 signaling to maintain the polyfunctionality of antigen-specific $\mathrm{T}$ cells. The precise mechanism is unclear and needs further investigation. Low-dose PD-1-laIL-2 effectively rejuvenates the effector function of PD- $1^{+}{ }^{+}$IIM $^{+}$TILs. This also suggests that PD-1 $1^{+}$TIM $^{+}{ }^{+}$TILs are more sensitive to IL-2 than other populations, such as Tregs, and reinforces our rationale that targeted delivery of low amounts of IL-2 to antigen-specific T cells enhances antitumor efficacy without causing side effects.

Overall, our data demonstrate that our next-generation PD-1-laIL-2 construct can carry the T cell growth factor IL-2 to tumor-specific $\mathrm{T}$ cells in a safe and effective manner. Our study also revealed a new target for tumor-specific $\mathrm{T}$ cells and the novel function of IL- 2 in reactivating PD-1 $1^{+} \mathrm{TIM}^{+} \mathrm{T}$ cells.

\section{Methods}

Mice. Eight-week-old WT BALB/c and C57BL/6 mice were purchased from The Jackson Laboratory. $\operatorname{Rag1}^{-{ }^{--}}\left(\operatorname{Rag}^{\mathrm{tm} 1 \mathrm{Mmom}} / \mathrm{J}\right)$ and NOD scid gamma mice were maintained internally. All mice were maintained 
under specific pathogen-free conditions. Animal care and experiments were carried out under institutional and National Institutes of Health protocols and guidelines.

Cell lines and reagents. A2O and Renca cells were purchased from the American Type Tissue Culture Collection (ATCC). MC38 cells were purchased from Kerafast. All cell lines were routinely tested using a mycoplasma contamination kit (R\&D Systems). A20 and Renca cells were cultured in RPMI 1640 medium supplemented with $10 \%$ heat-inactivated fetal bovine serum, $2 \mathrm{mmol} / \mathrm{L} \mathrm{L}$-glutamine, $0.1 \mathrm{mmol} / \mathrm{L}$ MEM nonessential amino acids, $100 \mathrm{U} / \mathrm{mL}$ penicillin, and $100 \mathrm{U} / \mathrm{mL}$ streptomycin under $5 \% \mathrm{CO}_{2}$ at $37^{\circ} \mathrm{C}$. MC38 cells were cultured in Dulbecco's modified Eagle's medium supplemented with $10 \%$ heat-inactivated fetal bovine serum, $100 \mathrm{U} / \mathrm{mL}$ penicillin, and $100 \mathrm{U} / \mathrm{mL}$ streptomycin under $5 \% \mathrm{CO}_{2}$ at $37^{\circ} \mathrm{C}$.

Anti-IL-2R $\beta$ (clone TM- $\beta 1$ ), anti-CD4 (clone GK1.5), anti-CD8 (clone 53-5.8), and anti-PD-1 (clone J43) were purchased from Bio X Cell. Anti-PD-L1 (atezolizumab) was provided by the UT Southwestern Simmons Cancer Center Pharmacy. FTY720 (fingolimod) was purchased from Selleck Chemicals. PD-1-laIL-2, PD-1-wtIL-2, ErblaIL-2, Erb-wtIL-2, and PD-L1-laIL-2 were produced in house by linking IL-2 to antibodies. Briefly, single-chain variable fragment of antibodies with Fc was cloned into pEE6.4 (Lonza). IL-2 was fused with Fc with a GGGGS linker and cloned into pEE6.4. Heterodimerization of Ab-IL-2 was achieved by the knobs-into-holes approach (52). Plasmids encoding Ab-Fc and IL-2-Fc were cotransfected into FreeStyle 293-F cells (Thermo Fisher Scientific). The supernatant containing fusion proteins was purified with Protein A affinity chromatography (Repligen) according to the manufacturer's instructions.

Tumor growth and treatment. A20 cells $\left(2-10 \times 10^{6}\right)$, Renca cells $(4 \times$ $\left.10^{5}\right)$, or MC38 cells $\left(1 \times 10^{6}\right)$ were injected subcutaneously into the backs of 8- to 10-week-old mice. The tumor volumes were measured along 3 orthogonal axes (a, b, and c) and were calculated as follows: tumor volume $=a b c / 2$. The mice were treated with intraperitoneal injections of $20 \mu \mathrm{g}$ of PD-1-laIL-2, PD-1-wtIL-2, Erb-laIL-2, Erb-wtIL-2, or PD-L1laIL-2. For $\mathrm{CD}^{+} \mathrm{T}$ cell or $\mathrm{CD}^{+} \mathrm{T}$ cell depletion experiments, $200 \mu \mathrm{g}$ anti-CD8 antibody or $200 \mu \mathrm{g}$ anti-CD 4 antibody was injected intraperitoneally 1 day before PD-1-laIL-2 treatment. Two hundred micrograms of anti-IL-2R $\beta$ antibody were used for IL-2 pathway blockade.

Flow cytometry. Single-cell suspensions of cells were incubated with anti-CD16/32 (anti-Fc $\gamma$ III/II receptor, clone 2.4G2) for 30 minutes and then stained with conjugated antibodies. All fluorescently labeled antibodies were purchased from BioLegend or BD Bioscience. Samples were analyzed on a CytoFLEX flow cytometer (Beckman Coulter Inc.), and data were analyzed using FlowJo software (TreeStar).

$T$ cell isolation. Tumor tissues were excised and digested with 1 $\mathrm{mg} / \mathrm{mL}$ collagenase A (Roche) and $0.5 \mathrm{mg} / \mathrm{mL}$ DNase I (Roche) at $37^{\circ} \mathrm{C}$ for 30 minutes and then passed through a $70-\mu \mathrm{m}$ cell strainer to remove large pieces of undigested tumor fragments. $\mathrm{CD}^{+} \mathrm{O}^{+}$cells were isolated with EasySep Mouse CD90.2 Positive Selection Kit II (STEMCELL Technologies Inc.) according to the manufacturer's instructions. $\mathrm{CD} 90^{+}$cells were then stained with surface marker antibodies.

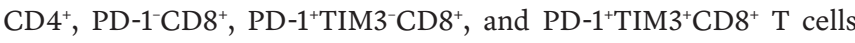
were sorted by BD FACSAria III (BD Biosciences).

Measurement of IFN- $\gamma$-secreting T cells by ELISPOT assay. dLN cells were resuspended in RPMI 1640 medium supplemented with 10\% FBS, $2 \mathrm{mmol} / \mathrm{L}$ L-glutamine, $100 \mathrm{U} / \mathrm{mL}$ penicillin, and $100 \mathrm{mg} / \mathrm{mL}$ streptomycin. A total of $3 \times 10^{5} \mathrm{dLN}$ cells was used for the assay. A20 cells were irradiated with a single dose of $60 \mathrm{~Gy}$. The ratio of A20 to immune cells was 1:4. After 48 hours of incubation, IFN- $\gamma$ production was determined with an IFN- $\gamma$ ELISPOT Assay Kit (BD Biosciences) according to the manufacturer's protocol. The visualized cytokine spots were enumerated with an ImmunoSpot S6 Analyzer (Cellular Technology Limited).

Single-cell RNA sequencing. Single-cell suspensions were loaded onto a Chromium Single Cell Chip (10x Genomics) according to the manufacturer's instructions for coencapsulation with barcoded gel beads at a target capture rate of approximately 10,000 individual cells per sample. Captured mRNA was barcoded during cDNA synthesis, and the barcoded cDNA was converted into pooled single-cell RNA-seq libraries for Illumina sequencing by using the Chromium Single Cell $5^{\prime}$ Library \& Gel Bead Kit (10x Genomics) according to the manufacturer's instructions. All samples were processed simultaneously with the Chromium Controller (10x Genomics) and the resulting libraries were prepared in parallel in a single batch. All libraries were sequenced with a NovaSeq 6000 sequencing system. Demultiplexing of sequencing results, barcode processing, read alignment, and unique molecular identifier (UMI) counting were performed using the 10x Cell Ranger analysis pipeline v6.0. Further quality control, feature selection, dimension reduction, unsupervised clustering, and differential expression analyses were performed using the Seurat R package v4.1.1 (53). Data are available at the Zenodo database (https://doi.org/10.5281/zenodo.5544128).

In vitro splenocyte activation. Splenocytes $\left(3 \times 10^{6} / \mathrm{mL}\right)$ were activated with anti-CD3 (BioLegend, $1 \mu \mathrm{g} / \mathrm{mL}$ ) and anti-CD28 (BioLegend, $1 \mu \mathrm{g} / \mathrm{mL}$ ) antibodies for 5 days. The cells were then subjected to surface marker staining and sorted with a BD FACSAria III (BD Biosciences).

Statistics. Data are shown as the mean \pm SEM. Statistical analyses were performed using a 2-tailed unpaired Student's $t$ test and Prism software (version 9.1, GraphPad Software). Two-way ANOVA was used to compare continuous outcomes across multiple experimental groups.

Study approval. This study was approved by the Institutional Animal Care and Use Committee of the University of Texas Southwestern Medical Center (animal protocol number [APN] 2015-101350, APN 2018-102474).

\section{Author contributions}

$\mathrm{ZR}, \mathrm{AZ}$, and YXF designed experiments, analyzed data, and wrote the manuscript. ZR and AZ developed the study methodology. ZR, AZ, ZS, YL, and JY performed experiments. JQ and BL provided reagents. ZR and YXF supervised the project.

\section{Acknowledgments}

The authors would like to thank all members of the Fu lab for their insightful feedback, as well as acknowledge the support of the UT Southwestern Medical Center Animal Research Center, the BioHPC Supercomputing Facility, and the Moody Foundation Flow Cytometry Facility. YXF holds the Mary Nell and Ralph B. Rogers Professorship in Immunology. This work was supported in part by Cancer Prevention and Research Institute of Texas grants RR150072 and RP180725 (to YXF) and RR170079 (to BL).

Address correspondence to: Zhenhua Ren or Yang-Xin Fu, Department of Pathology, UT Southwestern Medical Center, 5323 Harry Hines Boulevard, Dallas, Texas 75390, USA. Phone: 214.648.6539; Email: Zhenhua.Ren@UTSouthwestern.edu (ZR); Phone: 214. 648.6537; Email: Yang-Xin.Fu@UTSouthwestern.edu (YXF). 
1. Zhang L, et al. Intratumoral T cells, recurrence, and survival in epithelial ovarian cancer. $N$ Engl J Med. 2003;348(3):203-213.

2. Thomas NE, et al. Tumor-infiltrating lymphocyte grade in primary melanomas is independently associated with melanoma-specific survival in the population-based genes, environment and melanoma study. JClin Oncol. 2013;31(33):4252-4259.

3. Nishino M, et al. Monitoring immune-checkpoint blockade: response evaluation and biomarker development. Nat Rev Clin Oncol. 2017;14(11):655-668.

4. Hamid O, et al. Safety and tumor responses with lambrolizumab (anti-PD-1) in melanoma. N Engl JMed. 2013;369(2):134-144.

5. Topalian SL, et al. Safety, activity, and immune correlates of anti-PD-1 antibody in cancer. $N$ Engl JMed. 2012;366(26):2443-2454.

6. Brahmer JR, et al. Safety and activity of antiPD-L1 antibody in patients with advanced cancer. N Engl J Med. 2012;366(26):2455-2465.

7. Garon EB, et al. Pembrolizumab for the treatment of non-small-cell lung cancer. $N$ Engl J Med. 2015;372(21):2018-2028.

8. Herbst RS, et al. Atezolizumab for first-line treatment of PD-L1-selected patients with NSCLC. N Engl JMed. 2020;383(14):1328-1339.

9. Zou W, et al. PD-L1 (B7-H1) and PD-1 pathway blockade for cancer therapy: mechanisms, response biomarkers, and combinations. Sci Transl Med. 2016;8(328):328rv4.

10. Vilain RE, et al. Dynamic changes in PD-L1 expression and immune infiltrates early during treatment predict response to PD-1 blockade in melanoma. Clin Cancer Res. 2017;23(17):5024-5033.

11. Verma V, et al. PD-1 blockade in subprimed CD8 cells induces dysfunctional PD-1(+)CD38(hi) cells and anti-PD-1 resistance. Nat Immunol. 2019;20(9):1231-1243.

12. Kamada T, et al. $\mathrm{PD}-1^{+}$regulatory T cells amplified by PD-1 blockade promote hyperprogression of cancer. Proc Natl Acad Sci US A. 2019;116(20):9999-10008.

13. Sharma P, et al. Primary, adaptive, and acquired resistance to cancer immunotherapy. Cell. 2017;168(4):707-723.

14. Sun C, et al. Regulation and function of the PD-L1 checkpoint. Immunity. 2018;48(3):434-452.

15. Pitt JM, et al. Resistance mechanisms to immune-checkpoint blockade in cancer: tumor-intrinsic and -extrinsic factors. Immunity. 2016;44(6):1255-1269.

16. Schomburg A, et al. In vivo and ex vivo antitumor activity in patients receiving low-dose subcutaneous recombinant interleukin-2. Nat Immun. 1992;11(3):133-143.

17. Spolski R, et al. Biology and regulation of IL-2: from molecular mechanisms to human therapy. Nat Rev Immunol. 2018;18(10):648-659.

18. Taniguchi T, Minami Y. The IL-2/IL-2 receptor system: a current overview. Cell. 1993;73(1):5-8.
19. Ahmadzadeh M, Rosenberg SA. IL-2 administration increases CD4+ CD25(hi) Foxp3+ regulatory $\mathrm{T}$ cells in cancer patients. Blood. 2006;107(6):2409-2414.

20. Jensen $\mathrm{HK}$, et al. Increased intratumoral FOXP3-positive regulatory immune cells during interleukin-2 treatment in metastatic renal cell carcinoma. Clin Cancer Res. 2009;15(3):1052-1058.

21. Atkins MB, et al. High-dose recombinant interleukin 2 therapy for patients with metastatic melanoma: analysis of 270 patients treated between 1985 and 1993. JClin Oncol. 1999;17(7):2105-2116.

22. Rosenberg SA. IL-2: the first effective immunotherapy for human cancer. J Immunol. 2014;192(12):5451-5458.

23. Rosenberg SA. Interleukin 2 for patients with renal cancer. Nat Clin Pract Oncol. 2007;4(9):497.

24. Culliton BJ. FDA panel backs interleukin-2. Nature. 1992;355(6358):287.

25. Li Y, et al. Regulatory $\mathrm{T}$ cells control toxicity in a humanized model of IL-2 therapy. Nat Commun. 2017;8(1):1762.

26. Mott HR, et al. The solution structure of the F42A mutant of human interleukin 2. J Mol Biol. 1995;247(5):979-994.

27. Levin AM, et al. Exploiting a natural conformational switch to engineer an interleukin-2 'superkine'. Nature. 2012;484(7395):529-533.

28. Sun Z, et al. A next-generation tumor-targeting IL-2 preferentially promotes tumor-infiltrating CD8(+) T-cell response and effective tumor control. Nat Commun. 2019;10(1):3874.

29. Scheller J, et al. Immunoreceptor engineering and synthetic cytokine signaling for therapeutics. Trends Immunol. 2019;40(3):258-272.

30. Arenas-Ramirez N, et al. Interleukin-2: biology, design and application. Trends Immunol. 2015;36(12):763-777.

31. Johansson A, et al. License for destruction: tumor-specific cytokine targeting. Trends $\mathrm{Mol}$ Med. 2014;20(1):16-24.

32. Carnemolla B, et al. Enhancement of the antitumor properties of interleukin-2 by its targeted delivery to the tumor blood vessel extracellular matrix. Blood. 2002;99(5):1659-1665.

33. Weide B, et al. A phase II study of the L19IL2 immunocytokine in combination with dacarbazine in advanced metastatic melanoma patients. Cancer Immunol Immunother. 2019;68(9):1547-1559.

34 . Wagner K, et al. The targeted immunocytokine L19-IL2 efficiently inhibits the growth of orthotopic pancreatic cancer. Clin Cancer Res. 2008;14(15):4951-4960.

35. Klein C, et al. Cergutuzumab amunaleukin (CEA-IL2v), a CEA-targeted IL-2 variant-based immunocytokine for combination cancer immunotherapy: overcoming limitations of aldesleukin and conventional IL-2-based immunocytokines. Oncoimmunology. 2017;6(3):e1277306.

36. Kujawski M, et al. Potent immunomodulatory effects of an anti-CEA-IL-2 immunocytokine on tumor therapy and effects of stereotactic radiation. Oncoimmunology. 2020;9(1):1724052.

37. Mullard A. Restoring IL-2 to its cancer immunotherapy glory. Nat Rev Drug Discov. 2021;20(3):163-165.

38. Ribba B, et al. Prediction of the optimal dosing regimen using a mathematical model of tumor uptake for immunocytokine-based cancer immunotherapy. Clin Cancer Res. 2018;24(14):3325-3333.

39. Waldmann TA. The biology of interleukin-2 and interleukin-15: implications for cancer therapy and vaccine design. Nat Rev Immunol. 2006;6(8):595-601.

40. Dammeijer F, et al. The PD-1/PD-L1-checkpoint restrains $\mathrm{T}$ cell immunity in tumor-draining lymph nodes. Cancer Cell. 2020;38(5):685-700.

41. Siddiqui I, et al. Intratumoral $\mathrm{Tcfl} 1^{+} \mathrm{PD}-1^{+} \mathrm{CD} 8^{+} \mathrm{T}$ cells with stem-like properties promote tumor control in response to vaccination and checkpoint blockade immunotherapy. Immunity. 2019;50(1):195-211.

42. Ren Z, et al. CTLA-4 limits anti-CD20mediated tumor regression. Clin Cancer Res. 2017;23(1):193-203.

43. Sanmamed MF, Chen L. A paradigm shift in cancer immunotherapy: from enhancement to normalization. Cell. 2018;175(2):313-326.

44. Zheng $\mathrm{C}$, et al. Landscape of infiltrating $\mathrm{T}$ cells in liver cancer revealed by single-cell sequencing. Cell. 2017;169(7):1342-1356.

45. Sakuishi K, et al. Targeting Tim-3 and PD-1 pathways to reverse $\mathrm{T}$ cell exhaustion and restore anti-tumor immunity. J Exp Med 2010;207(10):2187-2194.

46. Duan Q, et al. Turning cold into hot: firing up the tumor microenvironment. Trends Cancer. 2020;6(7):605-618.

47. Sauve K, et al. Localization in human interleukin 2 of the binding site to the alpha chain (p55) of the interleukin 2 receptor. Proc Natl Acad Sci US A. 1991;88(11):4636-4640.

48. Ahn E, et al. Role of PD-1 during effector CD8 T cell differentiation. Proc Natl Acad Sci US A 2018;115(18):4749-4754.

49. West EE, et al. PD-L1 blockade synergizes with IL-2 therapy in reinvigorating exhausted T cells. JClin Invest. 2013;123(6):2604-2615.

50. Curiel TJ, et al. Specific recruitment of regulatory $\mathrm{T}$ cells in ovarian carcinoma fosters immune privilege and predicts reduced survival. Nat Med. 2004;10(9):942-949.

51. Zou W. Regulatory T cells, tumour immunity and immunotherapy. Nat Rev Immunol. 2006;6(4):295-307.

52. Zhang A, et al. Dual targeting of CTLA-4 and $\mathrm{CD} 47$ on $\mathrm{T}_{\mathrm{reg}}$ cells promotes immunity against solid tumors. Sci Transl Med. 2021;13(605):eabg8693.

53. Hao Y, et al. Integrated analysis of multimodal single-cell data. Cell. 2021;184(13):3573-3587. 